\title{
Soziotechnischer Wandel als graduelle Transformation
} \author{
$\begin{array}{ll} & \text { MPIfG Journal Article } \\ \text { Ulrich Dolata } & \text { Ulrich Dolata: Soziotechnischer Wandel als graduelle Transformation. In: Berliner Journal für Soziologie 21(2), 265 - 294 (2011). } \\ \text { Springer VS }\end{array}$ \\ The original publication is available at the publisher's web site: http://dx.doi.org/10.1007/s11609-011-0153-0 \\ The MPIfG Journal Articles series features articles by MPIfG researchers and visiting scholars published in peer-reviewed journals. \\ Max Planck Institute for the Study of Societies (MPIfG) Cologne | www.mpifg.de
}

\begin{abstract}
Zusammenfassung: Die durch neue technologische Möglichkeiten angestoßenen Prozesse soziotechnischen Wandels verlaufen nicht als radikale Brüche in kurzen Fristen, die schnell in eine neue Periode technologischer, institutioneller und organisationaler Kontinuität münden. Das, was nach zehn, zwanzig oder dreißig Jahren als radikaler soziotechnischer Umbruch erscheint, ist vielmehr das Ergebnis längerer Such- und Neustrukturierungsprozesse, die durch eine Vielzahl aufeinander bezogener technologischer und sozioökonomischer Veränderungen geprägt werden. Erst in ihrer Kumulation führen diese Veränderungen zu substanziellen Erneuerungen der technologischen, institutionellen und (inter-)organisationalen Grundlagen der Gesellschaft, der Wirtschaft oder eines Sektors. Wie lassen sich solche Prozesse eines ebenso radikalen wie sukzessiven Wandels analysieren? Über welche Formen vollziehen sie sich, welche Verlaufsmuster und Varianten können sie annehmen? Vor dem Hintergrund des durch neue Technologien angestoßenen Wandels von Wirtschaftssektoren wird in diesem Aufsatz ein Konzept gradueller soziotechnischer Transformation entwickelt, mit dem sich die vielschrittigen, oft erratischen und nichtlinearen Prozesse soziotechnischen Wandels analysieren und strukturieren lassen, die sich erst mit der Zeit zu substanziellen sektoralen Neuausrichtungen verdichten.
\end{abstract}

Schlüsselwörter: Neue Technologien · Internet · Gentechnik · Soziotechnischer Umbruch · Transformation $\cdot$ Pfadabhängigkeit $\cdot$ Adaptionsfähigkeit $\cdot$ Wirtschaftssektor

\section{Socio-technical change as gradual transformation}

\begin{abstract}
Processes of socio-technical change that are triggered by new technological opportunities do not occur as radical fractures over short periods of time, which then quickly lead to new periods of technological, institutional and organizational continuity. What appears to be radical socio-technical change is in fact the result of longer search and restructuring processes that are influenced by a number of related technological and socio-economic changes. Once these changes accumulate they lead to substantial adjustments within the technological, institutional and (inter-)organizational foundations of society, the economy or other sectors. How can an analysis of processes of both radical and gradual change be introduced? What modes are involved in their occurrence, which patterns do they follow and what variations do they assume? Against the background of technology-driven change within economic sectors, this paper develops a concept
\end{abstract}

(C) VS Verlag für Sozialwissenschaften 2011

U. Dolata $(\triangle)$

Institut für Sozialwissenschaften (SOWI VI), Universität Stuttgart,

Seidenstr. 36, 70174 Stuttgart, Deutschland

E-Mail: ulrich.dolata@sowi.uni-stuttgart.de 
of gradual socio-technical transformation. This concept can be used to analyze and structure multi-phased, often erratic and non-linear processes of socio-technical change that only over time evolve into substantial sectoral adjustments.

Keywords: New technologies $\cdot$ Internet $\cdot$ Genetic engineering $\cdot$ Socio-technical change $\cdot$ Transformation $\cdot$ Path dependency $\cdot$ Adaptability $\cdot$ Economic sector

\section{Le changement sociotechnique comme transformation graduelle}

Résumé: Les processus de changement sociotechnique entraînés par les nouvelles possibilités technologiques ne se produisent pas sous forme de ruptures soudaines et radicales inaugurant une nouvelle période de continuité technologique, institutionnelle et organisationnelle. Ce qui apparaît comme un bouleversement sociotechnique radical avec un recul de dix, vingt ou trente ans est plutôt le résultat de longs processus de recherche et de restructuration marqués par une multitude de mutations technologiques et socioéconomiques liées les unes aux autres. Ce n'est que par leur accumulation que ces mutations aboutissent à un renouvellement substantiel des fondements technologiques, institutionnels et (inter-)organisationnels de la société, de l'économie ou d'un secteur. Comment analyser les processus d'un changement aussi radical que progressif? Quels mécanismes sont à l'œuvre derrière eux, quelles formes typiques et quelles variations peuvent-ils présenter? Dans le contexte du changement entraîné par l'introduction de nouvelles technologies dans certains secteurs économiques, cet article développe un concept de transformation sociotechnique graduelle permettant d'analyser et de structurer les processus de changement sociotechnique progressifs qui se déroulent souvent de manière erratique et non linéaire et s'additionnent pour aboutir avec le temps à une réorganisation sectorielle substantielle.

Mots-clés: Nouvelles technologies · Internet · Génétique $\cdot$ Bouleversement sociotechnique · Transformation · Dépendance de sentier · Capacité d'adaptation - Secteur économique

\section{Einleitung}

Seit Ende der 1970er Jahre befinden wir uns in einer Periode eines grundlegenden soziotechnischen Umbruchs, in der die wissenschaftliche und technologische Basis der entwickelten kapitalistischen Gesellschaften in weiten Bereichen neu definiert wird. In ihrem Zentrum stehen ohne Zweifel die gesellschaftsweite Verbreitung und anhaltend radikale Weiterentwicklung digitaler Informations,- Kommunikations- und Vernetzungstechnologien. Daneben hat sich in dieser Zeit mit der Gentechnik ein zweites neues Technologiecluster etabliert, mit dem die gezielte Rekombination und Veränderung von Naturprozessen unterschiedlichster Art möglich wird.

Typisch für diesen großen soziotechnischen Umbruch ist zweierlei. Er lässt sich zum einen als markanter Einschnitt oder als Zäsur charakterisieren: als tiefgreifender Wandel der über längere Zeit vergleichsweise stabilen soziotechnischen Strukturen und Regeln, die weite Teile der Wirtschaft und Gesellschaft bis dahin geprägt haben. Er lässt sich zum anderen zugleich aber auch als zeitlich gestreckter und schrittweiser Prozess der Neustrukturierung beschreiben: als längere Periode, die geprägt wird durch eine anhaltend hohe Innovationsdynamik mit radikalen Neu- beziehungsweise Weiterentwicklungen von Wissen und Technologien sowie durch die damit einhergehende Suche nach dazu passenden Organisationsformen, Interaktionsmustern, Strukturen und Regeln. Das, was nach 
zehn, zwanzig oder dreißig Jahren als radikaler soziotechnischer Umbruch erscheint, ist das Ergebnis eines längeren Such- und Neustrukturierungsprozesses, der durch eine Vielzahl von mehr oder minder stark aufeinander bezogenen Veränderungen geprägt wird. Erst in ihrer Kumulation führen diese Veränderungen zu substanziellen Erneuerungen der technologischen, institutionellen und (inter-)organisationalen Grundlagen der Gesellschaft, der Wirtschaft oder eines Sektors.

Wie vollziehen sich solche langgestreckten soziotechnischen Umbrüche? Und welche Verlaufsformen können sie annehmen? Die Beantwortung dieser Fragen steht im Zentrum des folgenden Argumentationsgangs, in dem Prozesse einschneidenden soziotechnischen Wandels als graduelle Transformation konzeptualisiert werden.

In der innovationsökonomischen Literatur sind derartige Prozesse bereits Ende der 1980er Jahre als ,periods of mismatch“ bezeichnet worden: als länger andauernde Phasen der Suche nach, des Experimentierens mit und der interessegeleiteten Auseinandersetzung um neue Technologien sowie um veränderte Organisationsmuster, Strukturen und Regelungen, die zu ihnen passen (Freeman und Perez 1988; Dosi et al. 1988; Kitschelt 1991; ähnlich Dolata 1992, S. 215 ff.; Rip und Kemp 1998; Kemp et al. 2001). Derartige Einschätzungen, die den Ausgangspunkt der folgenden Überlegungen bilden, blieben allerdings vage in der Beantwortung der Fragen nach konkreten Verlaufsformen derartiger Umbruchperioden sowie nach typischen Mustern beziehungsweise Varianten, die diese annehmen können.

Die neuere soziotechnische Transformationsforschung ist da schon etwas konkreter. Sie versucht seit einigen Jahren, unterschiedliche „transition contexts“ zu identifizieren und - darauf aufbauend - alternative ,sociotechnical transition pathways“ herauszuarbeiten und voneinander abzugrenzen (Smith et al. 2005; Geels und Schot 2007; Geels und Kemp 2007; Dolata 2009). Die diesen Konzeptualisierungen zugrunde liegende Idee ist, dass sich soziotechnische Transformationsprozesse über das Zusammenspiel eines wie immer genau bestimmten technologischen Veränderungsdrucks und der wie immer genau bestimmbaren sozialen Möglichkeiten des untersuchten Feldes, diesen Druck wahrzunehmen, aufzugreifen und zu verarbeiten, vollziehen. Dies ist der erste Anknüpfungspunkt der folgenden Überlegungen.

Den zweiten Anknüpfungspunkt bildet die neuere Forschung zum institutionellen Wandel entwickelter kapitalistischer Gesellschaften, wie sie vor allem durch die Arbeiten von Kathleen Thelen und Wolfgang Streeck repräsentiert wird (Thelen 2003; Streeck und Thelen 2005; Mahoney und Thelen 2010). Dort wird ebenfalls betont, dass sich Prozesse gravierenden institutionellen Wandels in der Regel nicht als radikale Brüche darstellen lassen, sondern schrittweise erfolgen, längere Zeiträume in Anspruch nehmen und sich über distinkte Formen graduellen Wandels konkretisieren, durch die sich die bestehenden Regelungsstrukturen kapitalistischer Gesellschaften sukzessive verschieben und auf ein neues handlungsleitendes institutionelles Muster zubewegen.

Ich werde diese Überlegungen im Folgenden weiterentwickeln und vor dem Hintergrund des durch neue Technologien angestoßenen Wandels von Wirtschaftssektoren ein Konzept gradueller soziotechnischer Transformation begründen, mit dem sich die vielschrittigen, oft erratischen und nichtlinearen Prozesse soziotechnischen Wandels analysieren und strukturieren lassen, die sich erst über die Zeit zu substanziellen sektoralen Neuausrichtungen verdichten. 
Das Konzept wird in drei Schritten ausformuliert. Der nächste Abschnitt beginnt mit Eindrücken aus zwei empirischen Fällen, die an die Idee gradueller Transformation als der weithin typischen Verlaufsform des substanziellen soziotechnischen Wandels heranführen sollen: dem Wandel des Pharmasektors durch die Gentechnik und der durch das Internet ausgelösten Transformation des Musiksektors. Es folgt eine konzise Zusammenfassung und Bewertung neuerer Konzepte zum institutionellen Wandel, in deren Zentrum die Herausarbeitung von ,modes of gradual transformative change of modern politicaleconomic institutions" steht (Streeck und Thelen 2005, S. 2) (Abschn. 3). Sie bilden die Grundlage meiner eigenen Überlegungen, die in Abschn. 4 zu einem analytischen Konzept gradueller soziotechnischer Transformation ausgearbeitet werden. Dort wird konkretisiert, über welche Formen sich Prozesse substanziellen soziotechnischen Wandels vollziehen, welche Verlaufsmuster und Varianten sie annehmen können. In Abschn. 5 werde ich die Kernelemente des Konzepts zusammenfassen und Überlegungen dazu anstellen, wie es sich forschungspragmatisch handhaben lässt.

\section{Sukzessive Veränderungen und substanzieller Wandel: Empirische Hinführungen}

Ich beginne mit zwei kurzen empirischen Rekonstruktionen. Die beiden Fälle sollen an die Idee heranführen, dass sich auch ein gravierender sektoraler Wandel in aller Regel über sukzessive, oft erratische und zunächst uneindeutige, an verschiedenen Orten stattfindende Veränderungen Bahn bricht, die erst mit der Zeit als Kumulation zahlreicher Transformationsereignisse ihre substanziellen Wirkungen entfalten.

\subsection{Fall 1: Der Pharmasektor und die Gentechnik}

Die Gentechnik hat in den vergangenen dreißig Jahren vor allem im Pharmasektor einen gravierenden Umbruch angestoßen. Sie hat dessen technologisches Profil und seine Wissensbasis neu justiert - neben die chemische Synthese sind gentechnologische Methoden und Verfahren als wichtige neue Instrumente der Forschung und Produktion getreten. Sie hat die Pharmamärkte verändert, auf denen gentechnologisch hergestellte Therapeutika, Impfstoffe und Diagnostika seit Mitte der 1980er Jahre an Bedeutung gewonnen haben. Sie hat signifikante Neuausrichtungen der Industriestrukturen und sektoralen Interaktionsmuster angestoßen - insbesondere durch die Etablierung neuer Biotechnologiefirmen und durch eine außerordentliche Intensivierung der Kooperationsbeziehungen zwischen Pharmaunternehmen, Start-up-Firmen und Forschungseinrichtungen. Und sie hat schließlich substanzielle Veränderungen der regulativen Rahmenbedingungen des Sektors angestoßen, die sich vor allem in eigenständigen gentechnischen Rechtsbestimmungen und Richtlinien niedergeschlagen haben.

Der durch diese Prozesse geprägte sektorale Wandel erfolgte freilich nicht abrupt und in kurzer Frist, sondern in einem längeren Neustrukturierungsprozess, der Mitte der 1970er Jahre begann und bis weit in die 1990er Jahre hineinreichte (Dolata 1996, 2003; Henderson et al. 1999; Barben 2007). Und er war auch über die Zeit nicht geprägt durch eine radikale Ab- bzw. Auflösung des bestehenden soziotechnischen Profils, sondern 
durch selektive Revisionen, neuartige Kombinationen und substanzielle Erweiterungen der Leitorientierungen und Technologien, der Organisationsstrukturen und Interaktionsmuster, der strukturellen und institutionellen Rahmenbedingungen.

Den Ausgangspunkt bildete in der zweiten Hälfte der 1970er Jahre die Gründung biotechnologischer Start-up-Firmen in den USA, durch die die kommerzielle Erschließung der neuen Technologien angestoßen wurde. Die Pharmakonzerne selbst haben sich, von Ausnahmen abgesehen, zunächst zögerlich und tastend an dieses neue Technologiefeld angenähert und sich erst im Laufe der 1980er Jahre sukzessive strategisch und organisatorisch neu positioniert. Sie haben in diesem Jahrzehnt ihre auf Verfahren der chemischen Synthese ausgerichtete Forschung, Wirkstoffentwicklung und Produktion allerdings keineswegs vollständig revidiert, sondern schrittweise um gentechnologische Methoden und Verfahren erweitert. Und sie haben sich sukzessive auf für sie neue Modi kooperativer Forschung eingelassen und ihre bis dahin weitgehend inhouse-orientierten Forschungsund Entwicklungsaktivitäten um weitläufige Kooperationsgeflechte mit Start-up-Firmen und akademischen Forschungseinrichtungen ergänzt.

Zwischen Ende der 1970er und der ersten Hälfte der 1990er hat sich im Pharmasektor mit biotechnologischen Start-up-Firmen ein neuer Unternehmenstyp etablieren können, den es zuvor nicht gab. Diese Unternehmen haben sich im Laufe der 1980er Jahre zu wichtigen Innovationsträgern entwickelt und sind mit ihren flexiblen Forschungsstrukturen zu unabkömmlichen Kooperationspartnern der etablierten Pharmakonzerne geworden. Allerdings befanden sich 2006 unter den zwanzig umsatzstärksten Pharmakonzernen mit Amgen (Platz 14) und Genentech (Platz 19), das vollständig zu Hoffmann-LaRoche gehört, lediglich zwei Biotechnologieunternehmen (MedAdNews (13)9, Sept. 2007). Der weit überwiegende Teil der etwa 4000 Biotechnologiefirmen aus Nordamerika und Westeuropa ist demgegenüber über den Status von Zulieferern, Dienstleistern und forschungsintensiven Kooperationspartnern der Pharmakonzerne nicht hinausgelangt (Roijakkers und Hagedoorn 2006). Biotechnologiefirmen haben das Akteursspektrum und die Interaktionsformen des Sektors sukzessive erweitert, die klassischen Pharmakonzerne als dominierende Kernakteure allerdings nicht abgelöst.

Die Gentechnik hat nicht nur die Industriestrukturen und Interaktionsmuster des Sektors substanziell verändert. Sie hat auch neue Produkte hervorgebracht und die Pharmamärkte nachhaltig beeinflusst. $1982 \mathrm{kam}$ mit Humaninsulin ein erstes gentechnisch hergestelltes Therapeutikum auf den Markt. 25 Jahre später waren mehrere hundert Arzneimittel mit biotechnologisch gewonnenen Wirkstoffen weltweit auf dem Markt, von denen gut ein Dutzend als Blockbuster Umsätze zwischen einer und knapp vier Milliarden US-Dollar pro Jahr erzielten. Allerdings dominierten auch Mitte der 2000er Jahre herkömmliche, chemisch synthetisierte Therapeutika den weltweiten Arzneimittelmarkt. 2006 entfielen lediglich ca. $10 \%$ des weltweiten Pharmaumsatzes von damals $643 \mathrm{Mrd}$. US\$ auf Arzneimittel mit biotechnologisch gewonnenen Wirkstoffen. Biotechnologische Medikamente haben chemisch synthetisierte nicht verdrängt, sondern diese sukzessive ergänzt und das Produktportfolio erweitert (Dolata 2007). Das gilt gleichermaßen für die paradigmatischen Leitorientierungen des Sektors. Bio- und Gentechnologien sind als zentrale Methoden und Verfahren seit den 1980er Jahren vor allem aus der Pharmaforschung nicht mehr wegzudenken. Sie haben etablierte Forschungsmethoden und Produktionsverfahren wie Screening-Methoden und die chemische Synthese, die selbst einem dynamischen tech- 
nischen Wandel unterliegen, allerdings nicht abgelöst. Typisch sind vielmehr neuartige Kombinationen neuer mit alten Techniken (Drews 1999, S. 71 ff.).

Schließlich hat sich in den großen kapitalistischen Ländern und der Europäischen Gemeinschaft in Gestalt des Gentechnikrechts auch ein eigenständiger rechtlich-regulativer Rahmen für die Forschung, Entwicklung und Produktion herausgebildet, der neben das klassische Arzneimittelrecht getreten ist. Auch dieser Prozess zog sich, von Land zu Land mit unterschiedlichen Akzentuierungen, über einen längeren Zeitraum hin, der sich von Ende der 1970er bis weit in die 1990er Jahre erstreckte und von kontroversen politisch-gesellschaftlichen Debatten um den Sinn beziehungsweise die Ausgestaltung dieser neuen rechtlichen Rahmenbedingungen begleitet wurde. In Deutschland etwa setzten die entsprechenden Diskussionen bereits Ende der 1970er Jahre ein und mündeten erst 1990 in ein Gentechnikgesetz, das bereits 1993 substanziell novelliert wurde. Auch die parallel verlaufenden Aushandlungen im Rahmen der Europäischen Union konkretisierten sich erst 1990 in der Verabschiedung mehrerer Richtlinien, die den Umgang mit der Gentechnik in den Mitgliedstaaten regeln sollten (Schenek 1995; Bandelow 1999). Typisch für die Entwicklung des rechtlich-regulativen Rahmens für das neue Technikfeld war nicht bloß der lange Diskussionsvorlauf, sondern bis in die jüngste Vergangenheit auch seine wiederholte Neujustierung.

Insgesamt zeichnet sich der Transformationsprozess des Pharmasektors also nicht durch radikale Zerstörungen, die Auf- beziehungsweise Ablösung bestehender Kompetenzen, Technikprofile, Akteursfigurationen, Strukturen und Institutionen aus, sondern durch substanzielle Revisionen, Rekombinationen und Erweiterungen, die in ihrem Zusammenwirken den Sektor im Laufe der Zeit gleichwohl gravierend verändert haben.

\subsection{Fall 2: Der Musiksektor und das Internet}

Das Internet hat sich seit Mitte der 1990er Jahre als „multi-purpose technology“ in rasanter Geschwindigkeit zu einem neuen ubiquitären Informations-, Kommunikations- und Distributionsmedium entwickelt. Die klassischen Medienindustrien - die Musik- und Filmindustrie, der Buchhandel oder der Zeitungs- und Zeitschriftenmarkt - gehören zu den Bereichen, die durch das Internet und die Migration von digitalisier- und komprimierbaren Inhalten ins Netz in besonderer Weise herausgefordert werden. Die Musikindustrie war davon als erste betroffen. Das technologische Profil, die Märkte und die Distributionsmuster des Sektors verschieben sich seit einigen Jahren sukzessive von physischen Tonträgern hin zu digitalen Musikfiles, die nicht mehr über den stationären Handel, sondern über das Internet vertrieben werden. Die Interaktionsmuster, Konkurrenzbeziehungen und Machtstrukturen werden durch das Eindringen externer Akteure in den Sektor neu sortiert. Das Konsumverhalten vor allem jugendlicher Musikhörer hat sich mit der Möglichkeit des einfachen und nicht-marktlichen Tauschs digitaler Musik signifikant in Richtung kostenlosen „file sharings“ verändert. Unter diesen veränderten Bedingungen befinden sich etablierte wie neue kommerzielle Akteure auf der Suche nach wirtschaftlich tragfähigen Geschäfts- und Erlösmodellen rund um die digitale Musik sowie nach Anpassungen der Rechte- und Verwertungsbeziehungen an die neuen Gegebenheiten (Peitz und Waelbroek 2006; Tschmuck 2006). 
Obgleich der Wandel des Musiksektors eine außerordentliche Dynamik entfaltet und das Internet die Grundfesten des Sektors nachhaltig erschüttert haben, handelt es sich auch in diesem Fall um eine längere, wohl mindestens fünfzehn Jahre dauernde Phase der zwar krisenhaften und im Ergebnis radikalen, insgesamt jedoch schrittweisen Veränderung seiner technologischen, institutionellen und organisationalen Grundlagen, die Ende der 1990er Jahre einsetzte und gut ein Jahrzehnt später noch keineswegs abgeschlossen ist (Dolata 2008b; Burkart und McCourt 2006). Sie wurde bis Mitte der 2000er Jahre zunächst geprägt vom Aufschwung des nicht-marktlichen Musiktauschs über Musiktauschbörsen und massenhaften ,file sharings“ zwischen Musikkonsumenten, von ersten kommerziellen Download-Angeboten, insbesondere dem iTunes Store von Apple sowie von ebenso hilf- wie erfolglosen Versuchen der Musikkonzerne, ihr etabliertes Geschäft zu verteidigen und weitgehend unbeschädigt in das Internetzeitalter hinüberzuretten.

In der zweiten Hälfte des Jahrzehnts hat das Geschäft mit digitalen Musikfiles aus dem Internet erste Konturen angenommen und ist mittlerweile in signifikante Größenordnungen hineingewachsen. Allerdings verschieben sich die Umsatzanteile auf den großen Musikmärkten nicht schlagartig, sondern schrittweise von physischen Tonträgern (vor allem CDs) zu digitaler Musik (Downloads, Online-Abonnements, Klingeltöne). Trotz eines enormen Aufschwungs des Verkaufs von digitaler Musik entfielen 2008 auf physische Tonträger in den USA noch 59\% und in Deutschland 91\% des Jahresumsatzes (RIAA 2010; Bundesverband Musikindustrie 2010, S. 13). Auch wenn sich die Gewichte weiter zugunsten digitaler Musik verschieben werden, konnte bis dahin von einer radikalen Ablösung der CD durch Musikfiles noch keine Rede sein. Physische Tonträger und digitale Musik werden noch einige Jahre koexistieren und parallel vermarktet werden.

Seit Mitte der 2000er Jahre haben auch die Musikkonzerne damit begonnen, sich auf die Veränderungen ihres Tätigkeitsfeldes einzulassen, ihr Geschäft neu zu ordnen und mit neuen internetbasierten Geschäftsmodellen zu experimentieren - etwa mit der Parallelvermarktung von Tonträgern und Musikfiles, mit dem Verkauf von Musik über OnlineAbonnements, mit ihrer kostenlosen Bereitstellung und Refinanzierung über Werbung, Tourneen oder Handyverkäufe und mit der Komplettvermarktung der bei ihnen unter Vertrag stehenden Künstler (IFPI 2009). Diese organisationalen Revisionen erfolgten unter den Bedingungen großer Unsicherheit über die weitere Entwicklung des Musikgeschäfts und waren auch 2011 noch keineswegs abgeschlossen. Erst die kommenden Jahre werden zeigen, ob bzw. welche internetbasierten Geschäfts- und Erlösmodelle sich als tragfähig erweisen (IFPI 2011).

Die Kommerzialisierung digitaler Musik erfordert nicht nur das Austesten neuer Geschäftsmodelle und entsprechende organisationale Restrukturierungen, sondern auch eine weitreichende Neuordnung des Rechte- und Verwertungsrahmens, der bis in die Mitte der 2000er Jahre auf physische Tonträger zugeschnitten war. Solche institutionellen Neujustierungen sind ausgesprochen zeit- und abstimmungsaufwendig, da sie zwischen einer größeren Zahl relevanter Akteure mit zum Teil stark auseinandergehenden Interessen ausgehandelt, ausprobiert und abgestimmt werden müssen - zwischen den Musikunternehmen, den unter Vertrag stehenden Künstlern, den Gesellschaften für Aufführungs- und Vervielfältigungsrechte sowie den digitalen Musikanbietern im Internet. Auch die Herausbildung eines zu einem neuen Geschäft passenden Rechte- und Verwertungsrahmens erfolgt nicht als großer und einmaliger Wurf, sondern über schrittweise 
Veränderungen, die durch subversive Netzaktivisten immer wieder unterlaufen und zur Disposition gestellt werden (Rodriguez et al. 2007).

Schließlich wird der Umbruch des Musiksektors von Veränderungen seiner Akteursfigurationen, Konkurrenzverhältnisse und Machtstrukturen geprägt. Dabei gibt es zwangsläufig Gewinner und Verlierer, allerdings auch in diesem Fall nicht unbedingt einen radikalen Austausch der Spieler. Die Musikkonzerne und die großen stationären Handelsketten, die den Kern der ,alten“ Musikindustrie ausmachten, sind nach wie vor dabei - und haben seit Mitte der 2000er Jahre damit begonnen, ihr Geschäft auf die digitale Musik auszuweiten. Sie könnten als Produzenten, globale Promotoren, Rechteinhaber und etablierte Distributoren durchaus wichtige Akteure einer umstrukturierten Musikindustrie bleiben. Sie haben sich mittlerweile allerdings mit einer Reihe von starken neuen Akteuren auseinanderzusetzen, die sich nicht nur zu gleichberechtigten Verhandlungs- und Vertragspartnern der Musikkonzerne, sondern auch zu einer Herausforderung der großen stationären Handelsketten entwickelt haben. Diese kommen aus der Computerindustrie (Apple), der Telekommunikationsindustrie (T-Online), der Mobilfunk- und Handyindustrie (Vodafone, Nokia) oder aus dem Handel (Amazon, Wal-Mart), dominieren mit ihren Angeboten die internetbasierte Distribution digitaler Musik und sind die zentralen Treiber des netzbasierten Musikgeschäfts. Typisch ist auch in diesem Fall eine substanzielle Erweiterung des Akteursspektrums, die mit einer Neuordnung der Interaktionsmuster und Machtverhältnisse im Sektor einhergeht (Dolata 2008a).

Das alles weist auch den ohne Zweifel radikalen Umbruch der Musikindustrie als längeren Prozess der Neujustierung und -organisation des Sektors aus, der geprägt wird von einer Diversifikation der Musikvermarktung, der Herausbildung neuer Vertriebsformen, der Redefinition seiner institutionellen Rahmenbedingungen, der Ausdifferenzierung des ihn tragenden Akteursspektrums und den damit einhergehenden Veränderungen in den sektoralen Macht- und Einflussstrukturen.

\section{Zwischen Kontinuität und Bruch: Institutionalistische Angebote zur Beseitigung eines blinden Flecks}

Die hier skizzierten Beispiele sind typisch für Prozesse substanziellen sektoralen Wandels. Das, was für den Pharma- und Musiksektor vorgebracht wurde, ließe sich in ähnlicher Weise etwa auch für den Einbruch des Internets in andere Mediensektoren - Film, Buchhandel, Zeitschriften und Zeitungen (Currah 2006, 2009; OECD 2010; Schrape 2010, 2011; Heng 2006; e-business watch 2005b) -, für die Einführung von E-Commerce in verschiedene Bereiche des Handels (Riehm et al. 2003), für den Übergang von Groß- zu (vernetzten) Mikrocomputern in der Computerindustrie der 1980er Jahre (Bresnahan und Malerba 1999; Kenney und Curry 2001) oder für die in den 1980er und 1990er Jahren vollzogene Digitalisierung und Deregulierung von Telekommunikationsinfrastrukturen zeigen (Werle 1990; Schneider 2001; Mayntz 2009). Sektoraler Wandel durch Technik zeichnet sich offenbar nicht durch einmalige und eruptive Brüche in kurzen Fristen aus, die schnell in eine neue Phase der Stabilität mit dann eher marginalen weiteren Anpassungen münden. Er wird in aller Regel auch nicht geprägt durch die Sprengung, den Zusammenbruch oder einen radikalen Austausch der bestehenden Technologien, Struktu- 
ren, Institutionen und Akteure. Typisch sind vielmehr längere, ein oder zwei Jahrzehnte anhaltende Phasen der Diskontinuität und Neujustierung, in denen sich ein Sektor über eine Vielzahl von schrittweisen Transformationen auf ein neues dominantes soziotechnisches Design zubewegt, das zudem durch anhaltende technologische Innovationsdynamiken immer wieder erneut auf den Prüfstand gestellt wird.

Mit dichotomen Typisierungen, die lediglich zwischen langen Perioden der Stabilität und seltenen, durch exogene Schocks ausgelösten, ebenso abrupten wie radikalen Brüchen unterscheiden, lassen sich die realen Verläufe des technikinduzierten sektoralen Wandels nicht angemessen erfassen. Derartige Vorstellungen, die etwa in institutionalistischen und Pfadabhängigkeitskonzepten weit verbreitet sind, blenden das eigentlich interessante Feld zwischen Stabilität einerseits und radikalen Systembrüchen andererseits aus. ${ }^{1}$

Mittlerweile gibt es allerdings eine Reihe von Beiträgen, die sich von dieser einfachen Alternative zwischen radikalem Wandel und lang anhaltender Kontinuität gelöst und damit begonnen haben, das brachliegende Feld dazwischen zu vermessen (Thelen 2003; Streeck und Thelen 2005; Hall und Thelen 2009; Mahoney und Thelen 2010; Djelic und Quack 2003, 2007; Quack 2005; Campbell 2004, 2006; King 2007). Inhaltlich sind diese Beiträge zumeist weit vom hier verhandelten Thema entfernt. Sie bieten allerdings interessante konzeptionelle Hinweise, die für Untersuchungen technikinduzierten Wandels aufgegriffen und weiterentwickelt werden können.

Das gilt insbesondere für die von Kathleen Thelen entwickelte Idee gradueller institutioneller Transformationen und ihre Suche nach ,modes of gradual but nevertheless transformative change" (Streeck und Thelen 2005, S. 19; Thelen 2003; Mahoney und Thelen 2010). Den Hintergrund, vor dem sie und ihre Ko-Autoren entsprechende Überlegungen entwickelt haben, bildet der seit den 1980er Jahren beobachtbare institutionelle Wandel hochentwickelter kapitalistischer Gesellschaften in Richtung Liberalisierung. Eine erste wesentliche Eigenheit dieses Wandels ist, dass er sich über einen längeren Zeitraum erstreckt und eher durch eine Vielzahl schrittweiser, subtiler und sich verstärkender Veränderungen als durch abrupte Einschnitte oder radikale Brüche geprägt wird. „The current transformation of modern capitalism [...] unfolds by and large incrementally, without dramatic disruptions like the wars and revolutions that were characteristic of the first half of the twentieth century“ (Streeck und Thelen 2005, S. 4). Diese graduellen Transformationen sind in ihrer Anhäufung über die Zeit allerdings alles andere als unbedeutend geblieben. Sie haben - das ist die zweite wesentliche Eigenheit des Wandels - in ihrer Kumulation sukzessive zu substanziellen Veränderungen der institutionellen Grundlagen kapitalistischer Gesellschaften geführt. „Ongoing change and its accumulating results increasingly suggest that the current process of liberalization involves a major recasting of the system of democratic capitalism as we know it, issuing in a social order dissociated

1 Diese Vorstellung von Phasen langanhaltender Kontinuität, die periodisch von radikalen Umbrüchen unterbrochen werden, ist weit verbreitet in institutionalistischen und Pfadabhängigkeitskonzepten. Deren Kernaussage lautet: „Path-dependent equilibrium is periodically ruptured by radical change, making for sudden bends in the path of history“ (Pempel 1998, S. 3; auch: Krasner 1988). Damit werden die Hürden für institutionellen und strukturellen Wandel sehr hoch gelegt: Unterhalb radikaler Umbrüche gibt es im Grunde nur Kontinuität; Prozesse kumulativen graduellen Wandels sind so nicht analysier- und erklärbar (kritisch dazu Greenwood und Hinings 1996; Beyer 2006; Walgenbach und Meyer 2008). 
from fundamental assumptions of social integration and political-economic conflict resolution that underlay the construction of the postwar settlement after 1945“ (ebd., S. 5; zur Transformation des deutschen Kapitalismus ausführlich Streeck 2009, S. 31 ff.). Ähnlich wie in der hier verhandelten Frage des substanziellen soziotechnischen Wandels werden also nicht radikale Brüche in kurzer Frist als typisch für gravierende Transformationen heutiger kapitalistischer Gesellschaften herausgestellt, sondern ,a type of change that is slow and transformative at the same time" (Streeck und Thelen 2005, S. 15).

Um diese Art des Wandels genauer zu erfassen und abzubilden, ist es in einem ersten Schritt notwendig, typische Formen zu identifizieren, über die er sich vollziehen könnte: „modes of change going beyond the familiar but perhaps ultimately quite rare cases of institutional ,breakdown" or wholesale replacement" (Thelen 2003, S. 221). Kathleen Thelen und Wolfgang Streeck haben das versucht und auf der Basis empirischer Fallstudien fünf ihres Erachtens relevante Modi graduellen Wandels, über die sich mit der Zeit substanzielle institutionelle Veränderungen durchsetzen können, in die Diskussion eingeführt (Thelen 2003; Streeck und Thelen 2005, S. 18 ff.; Mahoney und Thelen 2010):

- Layering. Gravierender Wandel kann sich erstens vollziehen, indem neue Elemente an bestehende institutionelle Arrangements angefügt werden und durch ihren Bedeutungszuwachs deren Substanz sukzessive verändern. Etablierte Institutionen werden in diesem Fall nicht grundsätzlich infrage gestellt, sondern durch ihre Ergänzung und Erweiterung um neue Regeln, Normen und Orientierungen transformiert, die mit der Zeit gegenüber den bereits vorhandenen an Einfluss gewinnen.

- Conversion. Die Ziele, Funktionen und Zwecksetzungen vorhandener Institutionen können zweitens auch an neue Herausforderungen und veränderte Interessenlagen angepasst und entsprechend neu justiert werden. In diesem Fall vollzieht sich der institutionelle Wandel über die Veränderung der bestehenden Arrangements selbst und nicht über deren Erweiterung und Anreicherung um neue Elemente. ,Institutions designed with one set of goals in mind are redirected to other ends“ (Thelen 2003, S. 228).

- Displacement. Der institutionelle Rahmen eines Feldes oder Systems kann sich drittens auch dadurch ändern, dass zunächst unter- oder nachgeordnete Regelungen und Orientierungen, die an seinen Rändern entstanden sind, mit der Zeit an Bedeutung gewinnen, dominant werden und die alten sukzessive verdrängen. Der Wandel erfolgt dann nicht über die Erweiterung oder Veränderung der bereits etablierten Institutionen, sondern über den Bedeutungszuwachs alternativer institutioneller Arrangements, die quer zu den vorhandenen stehen und durch die Expansion neuer Akteure getragen werden.

- Drift. Etablierte Institutionen können viertens mit der Zeit an Relevanz verlieren, erodieren und verkümmern, weil Veränderungen politischer, ökonomischer und sozialer Rahmenbedingungen nicht rechtzeitig wahrgenommen worden sind und Neuausrichtungen an diese sich ändernden Bedingungen unterbleiben bzw. unzureichend bleiben. Gradueller Wandel heißt in diesem Fall sukzessiver Bedeutungsverlust bestehender Institutionen aufgrund von Anpassungsunfähigkeit.

- Exhaustion. Während im Fall von Drift die Institution, obwohl sie an Bedeutung verliert, formal bestehen bleibt, zeichnet sich diese fünfte Variante institutionellen 
Wandels schließlich durch den sukzessiven Kollaps und Zusammenbruch von Institutionen aus, deren Zwecke sich erschöpft haben und die obsolet geworden sind. Das ist kein Niedergang aufgrund von Anpassungsunfähigkeit; die betroffenen Institutionen sind schlicht überflüssig geworden.

\section{Radikaler Wandel als graduelle Transformation: Eigenheiten und Varianten soziotechnischer Umbrüche}

Mit diesen Modi lassen sich verschiedene Möglichkeiten substanziellen Wandels plausibilisieren, die nicht als radikale Brüche in kurzen Fristen daherkommen, sondern sich über eine Vielzahl von sukzessiven Veränderungen Bahn brechen. Das macht den Reiz und die schnelle (Zitations-)Karriere aus, die diese Überlegungen in den vergangenen Jahren genommen haben. ${ }^{2}$

Das ist es auch, was das Konzept für den hier verhandelten Zusammenhang interessant macht. Wenn man größere soziotechnische Umbrüche als Transformationsperioden begreift, die sich in aller Regel über einen Zeitraum von fünfzehn, zwanzig, zum Teil dreißig Jahren erstrecken, dann stellt sich umgehend die Frage, wie sie konkret verlaufen bzw. welche Formen und Varianten es gibt, über die sich mit der Zeit neue soziotechnische Realitäten herausbilden. Das geschieht offenkundig nicht als schneller und eindeutiger Zusammenbruch bestehender soziotechnischer Ordnungen und auch nicht in Form ihrer radikalen und reibungslosen Ablösung durch grundsätzlich neue technologische, organisationale und institutionelle Gegebenheiten.

Wie also verlaufen soziotechnische Transformationsperioden? Lassen sich typische Varianten analytisch voneinander unterscheiden? Das Konzept von Thelen und Streeck und die von ihnen ins Spiel gebrachten Formen graduellen Wandels liefern erste Hinweise zur Beantwortung dieser Fragen, die allerdings in mehrerlei Hinsicht unzureichend bleiben.

In dem hier interessierenden Zusammenhang geht es nicht bloß um die Transformation sozialer Institutionen, die bei Streeck und Thelen im Vordergrund stehen, sondern um das Zusammenspiel von weitreichenden technologischen Umbrüchen, deren (potenzielle) Radikalität außer Frage steht, und dadurch angestoßenen, ineinander verschränkten Prozessen strukturellen, institutionellen und organisationalen Wandels. Transforma-

2 Der Auflistung der verschiedenen Formen gradueller Transformation haftet allerdings ein Hauch von Beliebigkeit und Austauschbarkeit an. So wird im erwähnten Aufsatz von Mahoney und Thelen (2010) auf den Modus „exhaustion“ ohne weitere Begründung wieder verzichtet. Warum? Ausgesprochen (fahr-)lässig geht Streeck (2009, S. 15) mit den entwickelten Kategorien um: „We suggested a few more types of slow change, in particular ,displacement", ,drift", and ,exhaustion". Time will tell which of these will survive, and whether they were more than elaborations on the two original Thelen models." Was dann übrig bleibt, ist nicht mehr als eine empirische Plausibilisierung dafür, dass es zwischen Kontinuität und Bruch in der Tat etwas Drittes gibt: „In any case, they may be regarded as an attempt to describe in empirically grounded institutionalist language , dialectical ' tendencies in social institutions undermining themselves in the course of their normal operation - the opposite of path-dependent reproduction." 
tionsprozesse, wie sie hier zur Debatte stehen, reichen also deutlich über institutionelle Veränderungen eines Feldes hinaus. Und sie erfolgen auch nicht über weite Strecken schleichend und unmerklich (,,almost imperceptibly“; Streeck 2009, S. 15), sondern von Anfang an machtvoll und offenkundig, vorangetrieben durch das Aufkommen grundlegend neuer technologischer Möglichkeiten, die sich all ihren Dynamiken zum Trotz gleichwohl erst über die Zeit in substanziellen soziotechnischen Neuausrichtungen niederschlagen. Eine Fokussierung auf Prozesse eher inkrementellen institutionellen Wandels ist für das hier verhandelte Thema also in zweierlei Hinsicht zu eng. Die soziotechnischen Felder, deren Transformation hier interessiert, stehen 1) unter einem massiven, z. T. radikalen Veränderungsdruck, der sich 2) sowohl auf ihre technologischen Grundlagen als auch - damit untrennbar verbunden - auf ihre strukturellen, institutionellen und organisationalen Fundamente erstreckt.

Wenn eine Transformationsperiode insgesamt rekonstruiert und als Typ auf den Begriff gebracht werden soll, dann genügt auch der Rückgriff auf einen der genannten Modi graduellen Wandels zur Erklärung nicht. Es ist eher unwahrscheinlich, dass länger andauernde Transformationsperioden mit all ihren heterogenen Einflussfaktoren, ausgreifenden Suchvorgängen und interessegeleiteten Auseinandersetzungen allein oder auch nur vornehmlich durch eine der beschriebenen Formen graduellen Wandels geprägt und charakterisiert werden könnten. Wesentlich plausibler ist, dass in solchen Transformationsperioden mehrere Formen kombiniert auftreten, die sich wechselseitig verstärken oder auch miteinander konkurrieren können und deren Bedeutung und Beziehung zueinander sich überdies im Laufe der Zeit signifikant verändern kann. Erst über die spezifische Weise des Zusammenspiels verschiedener Formen graduellen Wandels lassen sich distinkte Varianten institutioneller oder, in unserem Fall, soziotechnischer Transformation als Typen herausarbeiten und voneinander unterscheiden. Derartige Kombinationen sind von den Protagonisten des Konzepts bislang nicht in Erwägung gezogen oder gar durchgespielt worden.

Eine letzte Sache kommt hinzu. Wenn substanzielle Veränderungen typischerweise als Ergebnis sukzessiver und kumulativer Prozesse begriffen werden, dann ist natürlich auch zu klären, was graduelle Transformation von dynamischer Reproduktion unterscheidet, wann also graduelle Transformation tatsächlich substanziell wird und systemverändernde Dimensionen annimmt - ,in other words, where flexible reproduction through adaptation ends and the replacement of one social order with another begins" (Streeck 2009, S. 16).

Ich werde nun diskutieren und präzisieren, wie sich substanzieller soziotechnischer Wandel als graduelle Transformation konzeptualisieren lässt. Zunächst lege ich dar, was ich unter gradueller Transformation als der meines Erachtens typischen Verlaufsform substanziellen Wandels verstehe (Präzisierung 1). Daran anschließend begründe ich, warum sich soziotechnische Transformationsperioden in aller Regel durch Kombinationen unterschiedlicher Formen oder Modi graduellen Wandels auszeichnen (Präzisierung 2). Vor diesem Hintergrund identifiziere ich vier typische Varianten gradueller Transformation, die sich aus spezifischen Kombinationen ergeben (Präzisierung 3). 


\subsection{Soziotechnische Umbrüche als graduelle Transformation}

Die Ausgangspunkte substanziellen Wandels sind in den hier vorliegenden Fällen nicht soziale Phänomene wie die von Thelen und Streeck in den Mittelpunkt gestellten Liberalisierungsdynamiken, sondern neue Technologien, genauer: paradigmatisch neue technologische Möglichkeiten, die sich nicht mehr umstandslos in die bestehenden Strukturen, Institutionen und Organisationsformen eines Sektors einfassen lassen, sondern weitreichende soziotechnische Neujustierungen provozieren. Dass diese sich sukzessive, über einen längeren Zeitraum vollziehen, hat sowohl technologische als auch soziale Gründe.

Größere technologische Umbrüche zeichnen sich zwar immer durch historisch identifizierbare Zäsuren oder Entwicklungssprünge aus - etwa durch den Wechsel von Groß- zu Mikrocomputern Ende der 1970er Jahre, durch die Digitalisierung von Telekommunikationsinfrastrukturen in der ersten Hälfte der 1980er Jahre, durch den Durchbruch gentechnischer Methoden und Verfahren in der zweiten Hälfte der 1970er Jahre oder durch den Aufschwung des Internets als neuem Informations- und Kommunikationsmedium seit der zweiten Hälfte der 1990er Jahre. Derartige Umbruchsituationen münden allerdings nicht in kurzen Fristen in neue und stabile technologische Entwicklungspfade.

Das hängt einesteils am ausgesprochen ambigen Charakter neuer technologischer Möglichkeiten in ihrer Formierungsphase. Oft ist zunächst unklar, was mit ihnen konkret zu machen ist. Es wird mit ihnen experimentiert, es werden geeignete Anwendungen gesucht, sie müssen erprobt und auf spezifische Gegebenheiten ausgerichtet, weiterentwickelt oder umgedeutet werden. Sie stimulieren zunächst den Aufbau von zum Teil großen Erwartungen, Versprechen und Visionen über künftige Verwendbarkeiten wie auch erste, nicht selten kontroverse Vorstellungen über mögliche bzw. notwendig erscheinende institutionelle und organisationale Anpassungen. Diese Vorstellungen werden in vergleichsweise offenen Such- und Experimentierprozessen verfeinert, erneuert, umgedeutet oder auch revidiert und verstetigen sich erst mit der Zeit zu neuen handlungsprägenden Leitbildern, die oft deutlich von den frühen Visionen und Erwartungen abweichen (van Lente und Rip 1998; Bender 2006). Sie konkurrieren darüber hinaus mit bestehenden und funktionierenden Technologien, die oft ebenfalls weiterentwickelt werden. Etablierte Technologien werden nicht einfach gegen neue ausgetauscht. Letztere gewinnen sukzessive an Relevanz, entwickeln sich aus Nischen schrittweise zu wirkmächtigen Alternativen und verändern das technologische Profil eines Sektors über zahllose Such- und Selektionsvorgänge (Levinthal 1998; Geels und Kemp 2007).

Typisch für neue Querschnittstechnologien - für die gesamte Palette moderner I\&KTechniken, die Biotechnologie oder die Nanotechnologie - ist darüber hinaus ihr auch nach ihrer Formierung anhaltend dynamisches und fluides Profil: Sie sind auch dann, wenn sie bereits in größerem Umfang praktisch genutzt und ökonomisch verwertet werden, nicht einfach fertig und einsatzbereit, sondern werden über längere Zeiträume immer wieder substanziell verändert und weiterentwickelt (Freeman 1994). Unterhalb sehr allgemeiner paradigmatischer Charakterisierungen - der Digitalisierung im Fall von I\&K-Technologien oder der gezielten Rekombination von Naturprozessen in der Biotechnologie - zeichnen sich neue Technologiefelder nicht durch frühe und einmalige Schließungsvorgänge aus, die einen neuen und stabilen technologischen Standard und Entwicklungspfad konstituieren, der als verlässlicher Rahmen für sozioökonomische und 
institutionelle Neustrukturierungen gelten könnte. Sie werden vielmehr geprägt durch länger anhaltende technologische Dynamiken, Unsicherheiten und Revisionen, neue Öffnungen und auch überraschende Sackgassen. Technologische „lock-ins“ und Pfadabhängigkeiten (David 1985) sind unter diesen Bedingungen, wie zahllose Beispiele aus der Computer- und Halbleitertechnologie, der Kommunikationstechnik, der Biotechnologie oder der Nanotechnologie zeigen, heute alles andere als dauerhaft und irreversibel. Wesentlich typischer sind temporäre „lock-ins“, die in schneller Folge durch die technologische Entwicklung wieder aufgebrochen und modifiziert werden (Beyer 2006).

Das gilt auch für die mit technologischen Umbrüchen einhergehenden strukturellen, institutionellen und organisationalen Veränderungen. Zwar lassen sich auch hier historische Zäsuren identifizieren, in denen die über längere Zeiträume stabilen sozioökonomischen Koordinaten von Sektoren infrage gestellt werden. Die IBM-dominierte Computerindustrie, der staatsmonopolistisch organisierte Telekommunikationssektor, der chemisch ausgerichtete Pharmasektor oder die oligopolistisch strukturierte Musikindustrie zeichneten sich über Jahrzehnte hinweg durch stabile soziotechnische Arrangements aus, die mit dem Aufkommen grundlegend neuer technologischer Möglichkeiten sukzessive erodiert sind.

Der dadurch angestoßene sozioökonomische Wandel verläuft allerdings auch dort, wo der Veränderungsdruck gravierend ist, nicht als drastischer Umbruch in kurzen Fristen, der dann wiederum durch eine Phase der Kontinuität abgelöst wird. Etablierte Sektoren weisen mit ihren strukturellen, institutionellen und organisationalen Gefügen in aller Regel eine hohe Stabilität auf und können schon aufgrund ihrer konfigurativen Komplexitäten nur schrittweise auf neue (technologische) Bedingungen ausgerichtet werden (North 1990; DiMaggio und Powell 1993; Hannan und Freeman 1984). Die durch neue technologische Möglichkeiten angestoßenen organisationalen Restrukturierungen, der Aufbau neuer Geschäftsfelder, größere Umorientierungen in Produktion, Vertrieb, Forschung und Entwicklung erfolgen tastend und schrittweise. Bestehende Märkte und Konkurrenzkonstellationen verändern sich ebenfalls nicht schlagartig, sondern sukzessive. Neue Akteure, die an den Rändern des Sektors entstehen oder von außen kommen, können sich oft zwar schnell zu ernstzunehmenden Herausforderern entwickeln, müssen ihre Rolle allerdings über die Zeit stabilisieren und bestätigen. Signifikante Verschiebungen in den Akteurs- und Machtfigurationen sind nicht das Ergebnis einmaliger Disruptionen, sondern nehmen über länger anhaltende Interessenauseinandersetzungen und Einflusskämpfe Gestalt an. Und auch die Institutionalisierung eines neuen Technikfeldes, seine regulative Einfassung und die Suche nach passenden Rechts- und Verwertungsrahmen sind ein abstimmungs- und zeitaufwendiger Vorgang, der sich über komplexe, von unterschiedlichen Interessen geprägte Aushandlungsprozesse zwischen einer größeren Zahl relevanter Akteure konkretisieren muss (Mokyr 2002; Werle 2005).

Entsprechende Neujustierungen erfolgen zudem oft nicht antizipativ, in Erwartung dessen, was technologisch kommen mag - keiner kann dies so recht wissen -, sondern erst in Reaktion auf einen zunehmenden und zunehmend unabweisbaren technologischen Veränderungsdruck. Und sie vollziehen sich auch nicht folgerichtig und reibungslos, sondern sind umstritten, provozieren kontroverse gesellschaftliche Debatten und konkretisieren sich in oft schwierigen und konfliktträchtigen Aushandlungs- beziehungsweise Konkurrenzprozessen zwischen heterogenen Akteuren (Dolata 2009). Hinzu kommt, 
dass sich ein Sektor im Wandel mit all den Neujustierungen, die dort passieren, nicht einfach schrittweise und nachholend an bereits stabile und nicht mehr infrage stehende neue technologische Gegebenheiten anpassen kann. Die neuen Technologien befinden sich selbst weiterhin in schneller Bewegung und stellen mit ihren Entwicklungsdynamiken die davon berührten Sektoren und deren Akteure über einen längeren Zeitraum immer wieder vor neue Herausforderungen.

Auch unter dem Druck radikal neuer technologischer Möglichkeiten nehmen Prozesse sektoraler Restrukturierung also Zeit in Anspruch, sind aufgrund der oft unbestimmbaren technologischen Dynamiken mit großen Unsicherheiten behaftet und sind angesichts der sehr unterschiedlichen Interessenlagen der involvierten Akteure zum Teil sehr umstritten und heftig umkämpft. Besonders anschaulich zeigt sich das in den komplizierten und langwierigen Aushandlungsprozessen, in denen rechtlich-regulative Rahmenbedingungen auf neue technologische Bedingungen ausgerichtet werden - zum Beispiel bei der Herausbildung des Gentechnikrechts in den 1980er und 1990er Jahren oder bei der Neuformulierung urheberrechtlicher Bestimmungen infolge der Digitalisierung und des Internets.

Die vermeintliche Paradoxie hat System. Obgleich größere soziotechnische Umbrüche, wie sie hier beschrieben wurden, in der Regel ausgesprochen dynamisch verlaufen, mit signifikanten Restrukturierungen einhergehen und im Ergebnis oft radikal sind, lassen sie sich - erste Präzisierung - angemessen nur als Periode gradueller Transformation rekonstruieren und abbilden: als bei aller Dynamik und allem Veränderungsdruck vielschrittige und zeitlich gestreckte Herausbildung neuer soziotechnischer Realitäten. Weitreichende Veränderungen bestehender Organisationsstrukturen, Regeln und Rahmenbedingungen sind nicht im Handstreich zu haben. Sie werden vielmehr geprägt durch unsichere, zunächst oft experimentelle und umkämpfte Such-, Selektions- und Neustrukturierungsvorgänge, über die sich erst mit der Zeit wesentlich andere, das untersuchte Feld in neuer Weise strukturierende technologische, institutionelle und organisationale Architekturen herausbilden.

\subsection{Graduelle Transformation als Kombination verschiedener Modi des Wandels}

Damit ist noch nichts über typische Varianten gesagt, über die sich derartige Perioden gradueller Transformation konkret vollziehen können. Die von Thelen und Streeck unterschiedenen fünf Modi graduellen Wandels lassen sich zwar als Ausgangsmaterial verwenden, um solche Varianten auch in Prozessen technikinduzierten sektoralen Wandels aufzuspüren. Sie müssen dazu allerdings sowohl auf das Thema als auch aufeinander bezogen werden.

Wenn technikinduzierter sektoraler Wandel untersucht wird, dann scheint für Sektoren, die sich durch eine geringe Aufnahmebereitschaft oder Adaptionsfähigkeit gegenüber neuen Technologien auszeichnen, am Beginn einer Transformationsperiode zunächst das charakteristisch zu sein, was dort als „drift“ bezeichnet wird: eine unterentwickelte Wahrnehmungs- und Anpassungsfähigkeit der etablierten Akteure und Institutionen gegenüber sich verändernden technologischen Rahmenbedingungen, gekoppelt mit einer beträchtlichen Veränderungsresistenz. Die bestehenden Strukturen, Institutionen und Akteure schlittern durch zögerliches beziehungsweise durch Nichthandeln in die Krise und ver- 
lieren an Bedeutung. Dadurch eröffnen sich Spielräume für alternative Handlungsorientierungen und für neue Akteure, die in den Sektor drängen und dessen Restrukturierung vorantreiben. Man kann Letzteres, die typisierten Formen des Wandels von Thelen und Streeck ergänzend, als Expansion (,expansion“) bezeichnen - als proaktiv betriebene Aufnahme und Entwicklung der neuen technologischen Möglichkeiten und als damit verbundene Herausbildung neuer, auf sie bezogener Akteursmilieus mit eigenen Interessen, Strategien, Institutionen und Strukturen.

Die Kernphase einer (sektoralen) Transformationsperiode, in der neue technologische Möglichkeiten eine nicht mehr hintergehbare Relevanz erlangen und in der die wesentlichen strukturellen, institutionellen und organisationalen Veränderungen erfolgen - diese Kernphase wird ganz wesentlich durch die ersten drei oben skizzierten Modi graduellen Wandels geprägt: durch die sukzessive Neudefinition organisationaler Handlungsorientierungen, kollektiver Regeln und sektoraler Leitorientierungen („conversion“), durch signifikante Verschiebungen in den Akteursfigurationen, Konkurrenz- und Kooperationsmustern, Macht- und Einflussverhältnissen, die den Sektor bis dahin geprägt haben (,displacement“) und/oder durch die Neujustierung bestehender Strukturen, Institutionen und Organisationen im Sinne ihrer Erweiterung um neue Bestandteile (,layering“). Schließlich gehen derartige Veränderungen immer auch - mal stärker, mal moderat - mit dem Niedergang bestehender Strukturelemente, Institutionen und Organisationen einher, die im Zuge der Transformation obsolet geworden sind und verschwinden (,exhaustion“").

All das zeigt bereits, dass einzelne Formen des Wandels für sich genommen lediglich verschiedene Möglichkeiten darstellen, über die sich Prozesse gradueller Transformation konkretisieren können. Sie sind dagegen nicht dazu geeignet, eine Transformationsperiode insgesamt zu charakterisieren und sie als Typ auf den Begriff zu bringen. Es ist ausgesprochen unwahrscheinlich, dass eine Transformationsperiode, die sich über ein oder zwei Jahrzehnte hinzieht, von einem Modus allein oder maßgeblich geprägt werden kann. Dazu sind die institutionellen und organisationalen Felder, um die es geht, zu komplex, die in den Wandel involvierten Akteure mitsamt ihren strategischen Zielsetzungen zu heterogen und die Transformationsverläufe selbst zu erratisch und zu verästelt. Wesentlich wahrscheinlicher ist, dass derartige Transformationsperioden ihre typische Signatur erst durch spezifische Kombinationen verschiedener Formen graduellen Wandels erhalten, die sich in der einen oder anderen Weise aufeinander beziehen und ineinander verschränken und deren Bedeutung sich im Laufe der Zeit zudem nachhaltig verschieben kann. Die in diesem Text vorgebrachten Beispiele zeigen allesamt, dass sich mehrere Formen gradueller Transformation in der Regel überlagern und dass sich ihre Bedeutung und ihr Verhältnis zueinander im Zeitablauf auch verändern.

Die Transformation des Musiksektors ist ein sehr offenkundiger Fall: Sie lässt sich nicht durch einen dominanten Mechanismus graduellen Wandels charakterisieren - etwa „displacement“, „layering“ oder „,conversion“ -, sondern wird geprägt durch verschiedene, zum Teil komplementär wirkende, zum Teil aber auch widerstreitende Modi, die jeweils von verschiedenen Akteuren präferiert und eingebracht werden. Die Transformation des Sektors wird nicht nur beeinflusst von radikalen Infragestellungen der etablierten Institutionen und Organisationen des Feldes und von der Entwicklung beziehungsweise Expansion grundlegender institutioneller und organisationaler Alternativen zum her- 
Abb. 1: Formen und Varianten gradueller Transformation
Formen graduellen Wandels

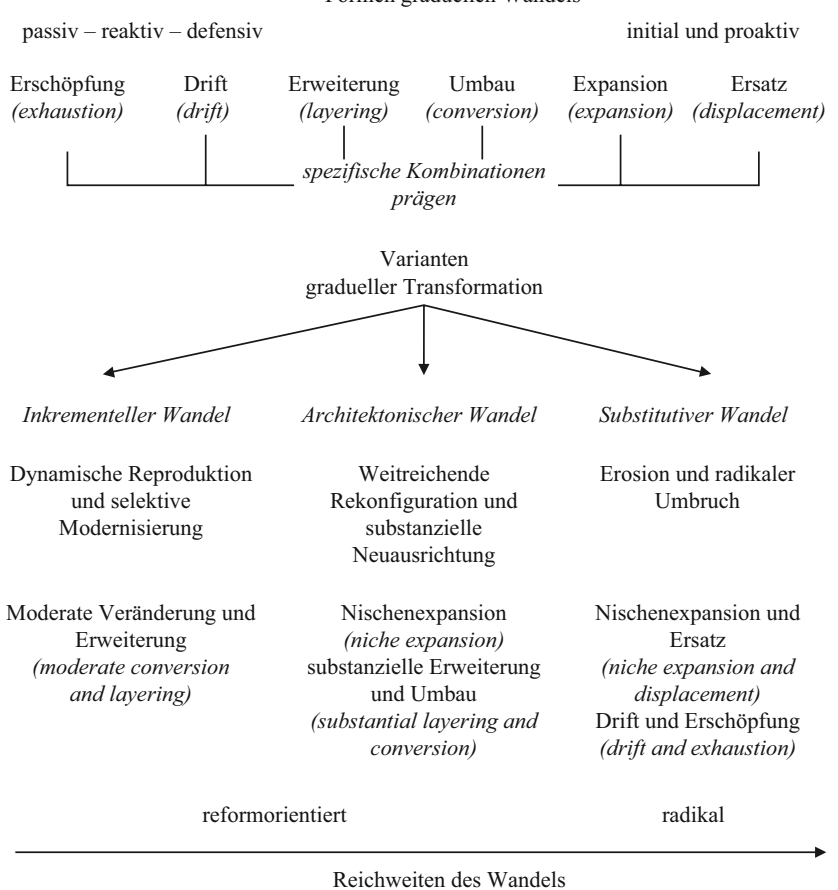

kömmlichen Musikgeschäft. Sie zeichnet sich zugleich durch Versuche der Etablierten aus, die durch die neuen Technologien ausgelösten Erschütterungen über moderate institutionelle und organisationale Veränderungen abzufedern - etwa über Novellierungen bestehender institutioneller Rahmenbedingungen oder Erweiterungen traditioneller Geschäftsfelder um neue Geschäftsmodelle.

Verallgemeinernd gesagt: Derart langgestreckte Neustrukturierungen komplex verfasster soziotechnischer Felder, wie sie hier im Mittelpunkt stehen, zeichnen sich im Laufe einer Transformationsperiode in der einen oder anderen Weise sowohl durch die Ausbildung und das Vordringen herausfordernder Alternativen (,expansion“ und „displacement") als auch durch substanzielle Weiterungen (,layering") sowie durch gezielte Veränderungen der bestehenden organisationalen Strukturen und Regelungsmuster (,conversion") aus, die überdies regelmäßig mit dem Bedeutungsverlust beziehungsweise dem Niedergang anpassungsunfähiger Organisationen und institutioneller Segmente („drift“ und „exhaustion“) einhergehen. Die Mischung macht's: Erst über das spezifische Zusammenspiel und die Gewichtung verschiedener Modi des Wandels erschließt sich ein Gesamtbild, die Signatur einer Transformationsperiode (s. Abb. 1).

Als zweite Präzisierung ist daher festzuhalten: In längeren Umbruchperioden vermengen und verschränken sich regelmäßig verschiedene Mechanismen graduellen Wandels in jeweils spezifischer Weise - und bilden erst durch ihr spezifisches Gewicht und ihre eigentümliche Kombination distinkte Varianten oder Muster aus, die eine Periode gradueller Transformation insgesamt als Typ auf den Punkt bringen können. Forschungspragmatisch gewendet heißt das: Die jeweils prägenden Formen graduellen Wandels sind 
fallweise zu identifizieren, zu gewichten und dynamisch, als sich über die Zeit verändernde Kombination aufeinander zu beziehen. ${ }^{3}$

\subsection{Varianten gradueller Transformation}

Je nachdem, welche Technologie und welchen Sektor man betrachtet, können die Bedingungen, die einer Transformationsperiode ihren Stempel aufdrücken, beträchtlich variieren. Dementsprechend unterscheiden sich unterhalb der Grundannahme, dass sich auch radikaler Wandel typischerweise als graduelle Transformation Bahn bricht, derartige Perioden, ihre Verläufe und Ergebnisse signifikant voneinander. Das wirft die Frage nach distinkten Varianten gradueller Transformation mit jeweils typischen Merkmalen auf, die ich nun in meiner dritten Präzisierung als stilisierte Typen vorstelle.

\subsubsection{Variante 1: Dynamische Reproduktion und inkrementeller Wandel}

Die erste Variante fällt aus dem Rahmen radikalen soziotechnischen Wandels. Sie ist charakteristisch für die Wirkungen grundlegend neuer Technologien, die eine relativ geringe sektorale Eingriffstiefe aufweisen. Die neuen technologischen Möglichkeiten ähneln in ihren sektoralen Wirkungen inkrementellen Innovationen, die bestehende Prozesse und Produkte zum Teil nachhaltig verbessern, ohne dass dazu größere soziotechnische Revisionen oder Restrukturierungen notwendig wären. Der technologisch bedingte Anpassungs- und Veränderungsdruck ist entsprechend moderat. Das gilt beispielsweise für die Nutzungsmöglichkeiten und die Integration internetbasierter Technologien in die Produktionsstrukturen und Kooperationsbeziehungen des Automobilsektors.

In solchen Fällen lässt sich die Einführung neuer Technologien als Prozess ihrer schrittweisen Implementation und weitgehenden Integration in bestehende Organisationen, Regeln und Strukturen analysieren, die in ihrem Kern nicht zur Disposition stehen. Sie wird maßgeblich getragen von den etablierten Akteuren des Sektors und erfolgt weitgehend im Rahmen der bestehenden Institutionen und Strukturen. Und sie wird geprägt von moderaten Veränderungen und Erweiterungen (,moderate conversion and layering“)

3 Auch Mahoney und Thelen (2010, S. 15) versuchen, verschiedene Typen institutionellen Wandels zu identifizieren - und greifen zu ihrer Erklärung auf zwei ihres Erachtens relevante Kontextbedingungen des Wandels zurück: auf die politischen Rahmenbedingungen, unter denen er stattfindet, und auf die Eigenheiten der Institution, die unter Anpassungsdruck steht. „Here the characteristics of both the political context and the institution in question together drive the type of institutional change we can expect. Political context and institutional form have these effects because they shape the type of dominant change-agent that is likely to emerge and flourish in any specific institutional context, and the kinds of strategies this agent is likely to pursue to effect change." Bei ihnen sind freilich die skizzierten Formen gradueller Transformation zugleich die wesentlichen Typen institutionellen Wandels - sie versuchen also nicht, verschiedene Modi miteinander zu kombinieren. Außerdem konzentrieren sie sich auf die Identifizierung von Akteuren, die den Wandel tragen und voranbringen (,,change-agents") - und lassen dabei all jene Akteure unberücksichtigt, die Prozesse des Wandels in ihrem Feld zu blockieren, zu verhindern und zu kanalisieren versuchen. 
vornehmlich der (inter-)organisationalen Strukturen, die mit dem Ziel der Modernisierung und Effizienzsteigerung betrieben werden.

Auch derartige auf neuen technologischen Möglichkeiten basierende Modernisierungsprojekte sind keineswegs triviale Vorgänge, wie etwa die Umstellung von Electronic-Data-Interchange-Systemen auf internetbasierte Kommunikationsstrukturen im Automobilsektor zeigt (e-business watch 2005a). Sie nehmen Zeit in Anspruch, sind aufwendig und organisationsintensiv und erfordern oft hohe Investitionen. Sie bringen natürlich auch spürbare Veränderungen mit sich, sonst würden sie nicht unternommen. Solche Veränderungen zielen allerdings nicht auf radikale sektorale Erneuerungen, sondern auf „performance improvements“ (Geels und Kemp 2007, S. 445), die auch für die Wirkungen inkrementeller Innovationen typisch sind.

Diese erste Variante gradueller Transformation lässt sich dementsprechend als Prozess dynamischer Reproduktion und inkrementellen Wandels beschreiben, der sich wesentlich unter Kontrolle der etablierten Akteure und im Rahmen bestehender und in ihrer Grundstruktur weitgehend stabil bleibender soziotechnischer Arrangements vollzieht.

\subsubsection{Variante 2: Substanzielle Neuausrichtung und architektonischer Wandel}

Das ist bei der zweiten Variante anders. Sie ist typisch für die Diffusion neuer eingriffstiefer Querschnittstechnologien in adaptionsfähigen Sektoren. Die neuen technologischen Möglichkeiten haben ein enormes sektorales Entwicklungs- und Einsatzpotenzial, das sich dort nur über substanzielle organisationale, strukturelle und institutionelle Veränderungen realisieren lässt. Ich nenne das große sektorale Eingriffstiefe neuer Technologien. In seiner Initialphase wird der Transformationsprozess in solchen Fällen oft zunächst durch Impulse von den Rändern beziehungsweise von außerhalb des Sektors geprägt, die durch neue bzw. sektorexterne Akteure angestoßen werden. Allerdings verhalten sich auch die etablierten Akteure des Sektors adaptiv. Sie lassen sich auch dann, wenn sie nicht zu den frühen Pionieren der Entwicklung und Nutzung der neuen Technologien zählen, vergleichsweise schnell auf die damit verbundenen neuen Möglichkeiten ein. Sie greifen sie aktiv auf, reorientieren ihre Aktivitäten entsprechend und richten ihre Forschung, Produktion und Organisationsstrukturen an den veränderten Bedingungen aus. Das bezeichne ich als hohe Adaptionsfähigkeit des unter Veränderungsdruck geratenen Sektors und seiner etablierten Akteure (Dolata 2008b).

Der Transformationsprozess wird unter diesen Bedingungen maßgeblich durch substanzielle und mehr oder minder geordnete Erweiterungen und Neugestaltungen (,substantial and orderly layering and conversion") des technologischen Profils, der Organisationsstrukturen und interorganisationalen Beziehungen, der Produkte und Märkte, der sektoralen Strukturen und institutionellen Rahmenbedingungen geprägt. Das technologische Profil des Sektors verändert sich vor allem durch Erweiterung und Rekombination mit der Zeit signifikant: Die etablierten Technologien müssen nicht abgelöst werden, sondern lassen sich mit den neuen Möglichkeiten produktiv verknüpfen. Das Akteursspektrum erweitert sich um neue Akteure, die nicht bloß in der Initialphase, sondern auch während der Institutionalisierung der neuen Technologien zu wichtigen Impulsgebern des sektoralen Wandels werden. Neue Formen interorganisationaler Kooperation werden ausprobiert, stabilisieren sich über die Zeit und binden die Newcomer weitgehend in die Neuordnung 
des Sektors ein. Die Ausrichtung und Arbeitsweise der Forschung und Entwicklung werden neujustiert, etablierte Produkte und Märkte werden nicht einfach ersetzt, sondern mit der Zeit um neue Varianten und Segmente erweitert. Schließlich werden auch die rechtlich-regulativen Rahmenbedingungen sukzessive an die neuen soziotechnischen Realitäten angepasst. Auch dies geschieht nicht in einem einmaligen Akt, sondern erfolgt mehrschrittig und angesichts anhaltender Innovationsdynamiken über einen längeren Zeitraum. Der durch die Gentechnologie angestoßene Wandel des US-amerikanischen Pharmasektors zwischen Mitte der 1970er und Mitte der 1990er Jahre ist ein solcher Fall (Henderson et al. 1999; Barben 2007).

Andere Modi sektoralen Wandels spielen demgegenüber eine untergeordnete Rolle. Vor allem angesichts der adaptiven Orientierungen der etablierten Akteure des Feldes bleiben „drift“ und „exhaustion“, also das durch Anpassungsunfähigkeiten geprägte Hineingleiten in eine Periode krisenhaften Umbruchs und die damit einhergehende Obsoleszenz maßgeblicher Akteure, Institutionen und Strukturelemente Randphänomene des Transformationsprozesses. Auch ein radikaler Austausch und Ersatz (,displacement“) bestehender Akteure, Institutionen und Strukturen ist weitgehend untypisch für diese Variante gradueller Transformation.

Insgesamt lässt sich der Transformationsprozess hier als substanzielle Neuausrichtung und architektonischer Wandel beschreiben, in dessen Verlauf das bestehende soziotechnische Gefüge des Sektors nicht durch ein grundlegend neues ersetzt, sondern nachhaltig erweitert und mit neuen Elementen (re-)kombiniert wird: „New regimes grow out of old regimes through cumulative adjustments and reorientations“" (Geels und Schot 2007, S. 407). Die neu entstehenden soziotechnischen Konstellationen fordern den etablierten Kern des Sektors heraus, allerdings ohne die ihn tragenden Akteure, Institutionen und Strukturen zu zerstören und ersetzen zu können.

\subsubsection{Variante 3: Erosion, radikaler Umbruch und substitutiver Wandel}

Die dritte Variante gradueller Transformation ist demgegenüber typisch für adaptionsunfähige Sektoren, in denen veränderungsresistente Akteure und Institutionen mit eingriffstiefen Technologien konfrontiert werden. Der eingangs skizzierte Einfall des Internets in den Musiksektor kann hierzu beispielhaft angeführt werden (Dolata 2008b).

Typischerweise kommen die neuen Technologien hier von außerhalb oder von den Rändern des Sektors. Ähnlich wie in der soeben beschriebenen Variante lassen sie sich vergleichsweise leicht aufgreifen, nutzen und weiterentwickeln - von wem auch immer. Allerdings treffen die neuen technologischen Möglichkeiten hier auf adaptionsunfähige Akteure und Institutionen. Gleichzeitig ist das bestehende technologische Profil des Sektors von Anfang an ausgesprochen anfällig gegenüber grundlegenden Innovationen und fällt als Schutzmechanismus gegen schnelle und substanzielle Veränderungen weitgehend aus. Die veränderungsresistenten Akteure und die institutionellen Gefüge, in denen sie sich bewegen, verlieren dadurch schnell an Kontrolle über den Transformationsprozess. Dadurch wird die Aneignung, Nutzung und Kommerzialisierung der neuen Technologien Newcomern überlassen, die den sektoralen Wandel nicht nur anstoßen, sondern auch dessen weiteren Verlauf wesentlich (mit)prägen können. 
Unter diesen Bedingungen wird der sektorale Transformationsprozess nicht nur in seiner initialen Phase, sondern darüber hinaus maßgeblich vorangetrieben durch die Expansion (,expansion“) neuer Akteure mit eigenen Interessen und Handlungsorientierungen, die eigene Strukturen und Regeln herausbilden können, welche quer zu den vorhandenen stehen (Leblebici et al. 1991; Flowers 2008). Sie zielen, mehr oder minder bewusst inszeniert, auf einen weitreichenden Ersatz und Austausch (,displacement") der den Sektor bis dahin prägenden Institutionen und Organisationen. Demgegenüber weigern sich die saturierten Akteure zunächst, im neuen Spiel aktiv mitzuspielen, versuchen, die neuen Entwicklungen zu blockieren, und reagieren erst zeitverzögert mit Formen systemkonformer Anpassung und Erneuerung. Sie schlittern zunächst durch Nichthandeln in eine für sie neue Situation, die sie nicht mehr kontrollieren (,drift“) und bemühen sich dann nachholend, über moderate Anpassungen, Erweiterungen und Veränderungen (,,moderate layering and conversion") ihrer organisationalen Profile und der institutionellen Rahmenbedingungen ins Spiel zurückzufinden. Im Laufe dieser Auseinandersetzung zwischen radikaler Erneuerung und moderater Veränderung, die den sektoralen Transformationsprozess in diesem Fall bestimmt, verlieren die bestehenden Akteure, Institutionen und Strukturen vor allem aufgrund ihrer Adaptionsdefizite signifikant an Bedeutung.

Auch in dieser Variante schälen sich neue Technologien, Produkte, Märkte, Akteure, Interaktions- und Regelungsmuster sukzessive - zunächst im Schatten der vorhandenen und zunächst weiterhin funktionierenden Konstellation - heraus. Sie gewinnen freilich schnell an Bedeutung, gehen mit dem Aufbau eigenständiger Regeln und Strukturen einher und lassen sich - dies ist der Unterschied zur zweiten Variante - nicht über rekonfigurierende Anpassungen und Erweiterungen weitgehend in die bestehenden sektoralen Zusammenhänge einbinden, sondern treten schnell in eine rivalisierende und substitutive Konkurrenz zu ihnen. Im Verlauf der Transformation führt dies aufgrund der zunehmenden Überlegenheit und Akzeptanz der neuen Technologien sowie der Adaptionsunfähigkeit des alten sektoralen Kerns zu einer radikalen Neuordnung und Neugewichtung der Akteursfigurationen, Institutionen und Strukturen des Sektors.

Im Vergleich zur zweiten Variante gradueller Transformation zeichnet diese sich also dadurch aus, dass konstitutive Bestandteile des organisationalen und institutionellen Gefüges mit der Zeit erodieren, obsolet werden (,exhaustion“) und durch neue Akteure, Interaktionsmuster, Institutionen und Strukturen abgelöst werden (,displacement“). Das lässt sich als radikaler Umbruch und substitutiver Wandel auf den Punkt bringen.

\subsubsection{Variante 4: Lang anhaltende Koexistenz, substitutiver oder architektonischer Wandel}

Typisch für die vierte und letzte Variante schließlich ist eine (Ausgangs-)Konstellation von hoher technischer und sozialer Stabilität und Persistenz, in deren Schatten sich sukzessive ein alternativer soziotechnischer Pfad als Nische mit eigenen Strukturmerkmalen, Regeln und Akteuren konstituiert und ausbreitet. Er wird allerdings nicht plötzlich zu einer existenziellen Herausforderung des etablierten sektoralen Kerns, sondern entwickelt sich über einen längeren Zeitraum unabhängig und parallel zum etablierten Pfad (Braun-Thürmann 2005, S. 46 ff.; Kemp et al. 2001). Das trifft vor allem für engmaschig vernetzte, kapital- und organisationsintensive großtechnische Infrastrukturen zu, wie sie 
z. B. im deutschen Energiesektor heute noch anzutreffen sind (Voß und Bauknecht 2007; Mautz et al. 2008; Praetorius et al. 2008). Je organisationsintensiver, technologisch komplexer und vermachteter die vorherrschenden soziotechnischen Systemstrukturen sind, desto voraussetzungsvoller und langwieriger ist deren radikale Veränderung.

Sektorale Transformationsprozesse werden in solchen Fällen zunächst über längere Zeit von Nischendynamiken geprägt, über die sich parallel zu und unabhängig von den bestehenden Systemstrukturen ein alternativer soziotechnischer Entwicklungspfad herausbildet, dessen Entwicklung oft durch politische Protektion und Intervention gefördert und geschützt wird - die Entwicklung erneuerbarer Energien ist ein solcher Fall. Die dominierende Form des Wandels lässt sich in dieser Phase als Expansion der Nische bezeichnen, durch die die Dominanz und Funktionslogiken der bestehenden sektoralen Kernstrukturen zunächst allerdings noch nicht herausgefordert und infrage gestellt werden. Es bildet sich eine anfangs sehr asymmetrisch verfasste Koexistenz zwischen unterschiedlichen Entwicklungspfaden heraus, die wenig miteinander gemein haben und bemerkenswert unabhängig voneinander existieren. Diese asymmetrische Koexistenz kann sich erst dann zu einer rivalisierenden Koexistenz entwickeln, wenn sich die Nische zu einer funktionierenden und weithin akzeptierten soziotechnischen Alternative sowie zu einer marktrelevanten Größe entwickelt hat.

Im weiteren Transformationsverlauf, der geprägt wird durch zunehmend direkte Rivalitäten zwischen dem Kern und der emanzipierten Nische, sind dann zwei unterschiedliche Entwicklungsrichtungen möglich.

Die Koexistenz kann zum einen in einen Prozess substitutiven Wandels übergehen - das ist die radikale Möglichkeit. Das dominante soziotechnische System und seine Akteure geraten in diesem Fall nicht nur durch überlegene und expandierende technologische Alternativen, sondern auch durch entsprechende politische bzw. gesellschaftliche Präferenzen und Weichenstellungen derart unter Druck, dass ihre Legitimation und Funktionsberechtigung erodieren. Die sukzessive Erschöpfung (,exhaustion“) der dominanten Technologien und der sie tragenden Institutionen und Akteure geht einher mit ihrem schrittweisen Ersatz (,displacement“) durch eine substanziell neue soziotechnische Konstellation - mit anderer technologischer Basis, anderen Institutionen und neuen bzw. strategisch grundlegend neu orientierten Akteuren, die sie tragen.

Alternativ denkbar und keineswegs unwahrscheinlicher ist ein Transformationsverlauf, der sich demgegenüber wiederum als architektonischer Wandel bezeichnen lässt. In solchen Fällen werden die neuen technologischen Möglichkeiten, die sich in der Nische herausgebildet und stabilisiert haben, gezielt vom unter Druck geratenen etablierten Kern des Sektors adaptiert. Die bestehende soziotechnische Konstellation löst sich nicht auf, sondern wird durch die selektive Aufnahme und Einbettung alternativer technologischer Möglichkeiten substanziell erweitert (,substantial layering“) und selektiv umgebaut (,selective conversion“). Substanzielle Erweiterung und selektiver Umbau meinen, dass mit der Transformation neue (Teil-)Märkte entstehen, das Produktportfolio ergänzt wird, organisationale Strukturen daran ausgerichtet und bestehende institutionelle Arrangements an die veränderten soziotechnischen Bedingungen angepasst werden. Auch die damit verbundenen Veränderungen können über die Zeit durchaus weitreichend sein. Sie markieren allerdings keinen radikalen Bruch mit den bestehenden Technologien, Institutionen und Akteuren des Sektors, sondern zielen auf deren Reform und Rekonfiguration. 
Welche dieser beiden Transformationsmöglichkeiten großtechnischer Systeme - substitutiver oder architektonischer Wandel - sich letztlich durchsetzt, ist keineswegs nur oder vornehmlich das Ergebnis von Marktprozessen. Sektorale Machtkonstellationen sowie die Entfaltung politischen und gesellschaftlichen Veränderungsdrucks können hier auch eine wichtige Rolle als Einflussfaktoren des Transformationsverlaufs spielen. In jedem Fall zeichnet sich diese Variante durch besonders langwierige Prozesse gradueller Transformation aus, die sowohl die Phase des Nischenwachstums technologischer Alternativen als auch die Phase ihrer rivalisierenden Koexistenz mit der bestehenden soziotechnischen Konstellation betrifft.

\section{Graduelle Transformation als forschungspragmatisches Konzept}

Mit dem hier vorgestellten Konzept gradueller Transformation lassen sich die konkreten Verlaufsformen und Varianten größerer soziotechnischer Umbrüche, die zunächst eher unbestimmt als ,period of mismatch“ (Dosi et al. 1988, S. 11) oder auch als ,period of considerable confusion“ (Henderson und Clark 1990, S. 12) beschrieben worden sind, genauer analysieren. Die ,,black box“ wird geöffnet: Der Blick richtet sich auf die spezifischen Einflussfaktoren, Abläufe und Dynamiken derartiger Umbruchperioden, die nun als langgezogene und vielstimmige, oft umstrittene und umkämpfte Such-, Selektionsund Neujustierungsvorgänge nicht mehr nur bezeichnet, sondern auch präzise rekonstruiert und kategorial abgebildet werden können. Sie werden geprägt von

- der sukzessiven Diffusion neuer Technologien, deren Eigenheiten sich noch im Laufe des Transformationsprozesses zum Teil gravierend verändern können;

- der schrittweisen Herausbildung daran ausgerichteter neuer Märkte und nicht-marktlicher Beziehungen, Konkurrenz- und Kooperationsmuster;

- der allmählichen Erneuerung der Strukturen und institutionellen Gefüge des beobachteten Feldes sowie

- der damit einhergehenden Veränderung der vorhandenen Akteursfigurationen, Machtund Einflussbeziehungen.

Transformation heißt: Im Ergebnis radikale Neuausrichtung eines Feldes, durch die sich sowohl dessen technologisches Profil als auch - damit verbunden - dessen soziale Koordinaten substanziell verändern. Graduell betont demgegenüber die wesentliche prozessuale Eigenheit derartiger Umbrüche, die sich grundsätzlich schrittweise, als Kumulation zahlreicher Transformationsimpulse vollziehen und über einen längeren Zeitraum erstrecken.

Das hier vorgestellte Konzept geht freilich über die allgemeine Aussage hinaus, dass sich auch substanzieller soziotechnischer Wandel typischerweise als graduelle Transformation vollzieht. Es liefert darüber hinaus die konkrete Handhabe, wie sich solche Umbruchperioden analysieren und einordnen lassen. Mit Hilfe der beschriebenen Formen graduellen Wandels, präziser: über relevante Kombinationen verschiedener Modi, können distinkte Transformationspfade herausgearbeitet und typische Muster gradueller Transformation identifiziert werden. Dabei lassen sich zwei idealtypische Kombinationen, die 
dem Transformationsprozess in unterschiedlicher Weise ihren Stempel aufdrücken können, voneinander unterscheiden.

Die erste Kombination ist Erweiterung - Umbau (layering - conversion). Auch wenn die wesentlichen Ausgangsimpulse des Wandels von den Rändern des Sektors oder von außerhalb kommen, werden sie in diesem Fall vom etablierten Kern vergleichsweise früh und proaktiv aufgegriffen. Der Transformationsprozess vollzieht sich vornehmlich über strategische Neuorientierungen der etablierten Akteure, als signifikante Erweiterung und Umgestaltung vorhandener Organisationsmuster, Institutionen und Strukturen, in die neue Elemente und Akteure eingefügt und mit den weiterbestehenden auf neue Weise kombiniert werden. Das ist die reformorientierte Variante gradueller Transformation, die nicht auf Substitution, sondern - je nachdem, wie eingriffstief die neuen Technologien sind - auf inkrementelle Anpassungen oder weiterreichende architektonische Veränderungen hinausläuft und für adaptionsfähige Sektoren typisch ist.

Alternativ dazu steht die zweite Kombination Expansion - Ersatz (expansion - displacement). Die Schwäche etablierter Akteure, sich auf grundlegend neue Technologien einzulassen, sie aufzugreifen und $\mathrm{zu}$ adaptieren, korrespondiert in diesem Fall mit einem signifikanten Bedeutungszuwachs avantgardistischer Akteure, die den sektoralen Umbruch durch ihre Aktivitäten nicht nur anstoßen, sondern auch im weiteren Verlauf dessen wesentliche Träger und Impulsgeber bleiben. Ihre Expansion geht einher mit dem Aufbau alternativer Regelungsmuster und Strukturen, die quer und in Konkurrenz zu den bereits vorhandenen stehen, diese sukzessive destabilisieren und mit der Zeit weitgehend ersetzen. Das ist die radikale Variante gradueller Transformation, die auf eine weitreichende Erosion und Substitution der bestehenden Organisationen, Strukturen und Institutionen hinausläuft und für adaptionsunfähige Sektoren typisch ist.

In beiden Varianten gradueller Transformation findet sich in unterschiedlicher Ausprägung auch die dritte Kombination Drift - Erschöpfung (drift - exhaustion). Für die radikale Variante ist sie konstitutiv. Die Expansion neuer Akteure und der Aufbau alternativer institutioneller Arrangements und Strukturen gehen hier einher mit der Anpassungsunfähigkeit der etablierten Akteure. Die bestehenden Institutionen und Strukturen werden obsolet und im Laufe des Transformationsprozesses sukzessive durch wesentlich andere abgelöst. Nur so macht „exhaustion“, also Obsoleszenz und Auflösung als Modus des Wandels überhaupt Sinn: Tragende Institutionen, Strukturen und Akteure werden nur dann wirklich überflüssig, wenn Neues bereitsteht und sie zu ersetzen vermag.

Auch die reformorientierte Variante gradueller Transformation geht immer einher mit dem Niedergang einzelner Akteure, die dem Veränderungsdruck nicht standhalten, sowie mit sektoralen Erneuerungsprozessen, durch die Teilbereiche des bestehenden institutionellen und strukturellen Gefüges an Bedeutung verlieren und ersetzt werden. Das ist in dieser Variante allerdings eher eine mitlaufende Begleiterscheinung architektonischer Veränderungen, die durch Erweiterungs- und Umgestaltungsprozesse bestehender Arrangements geprägt werden und in deren Umfeld natürlich immer auch einzelne Regelungsbereiche und Strukturelemente fallen gelassen und ersetzt werden.

Im konkreten Prozess soziotechnischer Transformation lassen sich die hier vorgestellten Formen und idealtypischen Kombinationen graduellen Wandels regelmäßig allesamt auffinden - allerdings in jeweils unterschiedlicher Mischung und Gewichtung. Die durch Erweiterung (layering) und Umbau (conversion) geprägte reformorientierte Variante wird 
natürlich auch beeinflusst durch die Expansion neuer Akteure und den Ersatz vorhandener Regeln und Strukturelemente. Umgekehrt laufen in der durch Expansion und Ersatz (displacement) geprägten radikalen Variante immer auch Versuche mit, Erosionsprozesse über moderate Erneuerungsinitiativen aufzuhalten bzw. zu kanalisieren.

Eine letzte wichtige und zugegeben schwer zu beantwortende Frage bleibt: Wann schlagen derartige Prozesse gradueller Transformation in neue Systemqualitäten um? Wann werden die vielen technologischen und sozioökonomischen Veränderungen tatsächlich substanziell und lösen jene „,cumulative commitments“ (Pierson 2000, S. 76) $\mathrm{ab}$, die den Sektor bis dahin geprägt, seine Stabilität und Reproduktion garantiert haben?

Während sich der Beginn einer soziotechnischen Transformationsperiode noch vergleichsweise klar festmachen lässt, sind eindeutige Umschlagpunkte im Laufe des Transformationsprozesses selbst erheblich schwerer zu identifizieren. Das wesentliche Charakteristikum gradueller Transformation ist ja gerade, dass sich neue, substanziell andere sektorale Bedingungen und Akteure nicht abrupt und in kurzer Frist herausbilden, sondern sukzessive, als Kumulation zahlreicher Ereignisse entstehen und sich erst mit der Zeit stabilisieren. Selbst exogene Schocks wie etwa der Einbruch des Internets in die bis dahin festgefügte Welt der Musikindustrie sind lediglich Ausgangspunkte einer Umbruchperiode und stoßen all jene Such-, Selektions- und Neujustierungsvorgänge an, die ihren Verlauf prägen und ihre Ergebnisse vorbereiten.

All das ändert natürlich nichts daran, dass sich im Prozess gradueller Transformation wichtige sektorale Koordinaten mit der Zeit signifikant verschieben und sich ein neues tragendes Gefüge des Sektors herausbildet. Wann kann davon gesprochen werden? Allgemein formuliert ist das dann der Fall, wenn sich sowohl das technologische Profil des Sektors als auch seine sozioökonomischen Grundlagen - die Institutionen und Strukturen - nicht nur substanziell verändert, sondern sich darüber hinaus als neue, zumindest im Grundsatz nicht mehr reversible soziotechnische Realität derart gefestigt haben, dass sie handlungsleitend für seine Akteure und konstitutiv für die Reproduktion(sfähigkeit) des Sektors insgesamt geworden sind. Dann kann von der relativen Stabilisierung einer neuen bzw. neu arrangierten sektoralen Regelungsstruktur gesprochen werden.

Die neuen Technologien, um die es geht, müssen dazu die alten nicht abgelöst haben. Sie müssen sich aber als nicht mehr hintergehbare neue Realitäten im Sektor festgesetzt haben. In von Fall zu Fall unterschiedlicher Gewichtung heißt das:

- Sie haben sich als unabkömmliche Basis neuer Forschungsmethoden, Produktionsverfahren oder Vertriebsstrukturen etabliert, ohne die die Reproduktion des Sektors nicht mehr gewährleistet werden kann;

- sie haben sich in Form neuer Angebote aus experimentellen Nischen emanzipiert, sind zu einer festen Marktgröße mit relevanten Umsatzanteilen geworden und bestimmen die künftigen Strukturierungen und Dynamiken der sektoralen Märkte;

- sie üben einen prägenden Einfluss auf die Strukturierung der privaten Nachfrage, auf Konsummuster und lebensweltliche Zusammenhänge aus.

Darüber hinaus muss die Suche nach dazu passenden Organisations-, Interaktions- und Regelungsmustern so weit fortgeschritten sein, dass daraus neue verbindliche und handlungsprägende sozioökonomische Rahmenbedingungen im Sektor entstanden sind und 
sich institutionalisiert haben, die sich nicht einfach wieder rückgängig machen und auflösen lassen. Dazu gehören (wiederum in von Fall zu Fall verschiedener Ausprägung):

- die Etablierung neuer sektoraler Kernakteure, die sich aus ihrem Nischendasein emanzipiert, als konstitutive Bestandteile des neu strukturierten organisationalen Feldes gefestigt und als Träger des Innovationsprozesses behauptet haben;

- die Festigung veränderter, an den neuen Technologien ausgerichteter Organisationsmuster bei den verbliebenen traditionellen Akteuren des Sektors, die ihren Neuorientierungs- und Umstrukturierungsprozess weitgehend abgeschlossen haben;

- die Stabilisierung neuartiger, zuvor nicht vorhandener kompetitiver wie kooperativer Interaktionsbeziehungen zwischen den beteiligten Akteuren, die den Austausch zwischen ihnen nicht mehr bloß fallweise und situativ regeln, sondern konstitutiv geworden sind;

- schließlich die Institutionalisierung wesentlich anderer Regeln - Recht, Normen, Standards -, die das Handeln auf neuer Grundlage strukturieren und die Reproduktionsbedingungen des betreffenden Sektors nunmehr prägen.

Im Pharmasektor beispielsweise war ein solcher Umschlagpunkt etwa Mitte der 1990er Jahre erreicht. Die pharmazeutische Forschung und Entwicklung war nun ohne die konstitutiven Beiträge neuer biotechnologischer Methoden nicht mehr denkbar, Biotechnologiefirmen hatten sich als neuer Unternehmenstyp im Sektor stabilisiert und das organisationale Feld erweitert, neue Formen der Kooperation zwischen den großen Pharmaunternehmen, Biotechnologie Start-ups und akademischen Forschungseinrichtungen hatten sich als nicht mehr hintergehbares Interaktionsmuster etabliert, die pharmazeutischen Märkte wurden durch gentechnisch gewonnene Medikamente, Impfstoffe und Diagnostika (mit)geprägt, ein neuer rechtlich-regulativer Rahmen für die gentechnologische Forschung und Produktion hatte sich sowohl in nationalen Zusammenhängen als auch im Rahmen der Europäischen Union und internationaler Regime herausgebildet (Dolata 1996, 2003; Barben 2007).

Relative Stabilisierung eines neuen sektoralen Regulationsmusters heißt also nicht Ersatz alter durch neue Technologien und Ablösung alter durch neue Akteure, Interaktionsmuster, Institutionen und Strukturen. Entscheidend ist vielmehr, dass im Zuge gradueller Transformation sowohl der Entwicklungsstand und die Nutzung der neuen Technologien als auch die damit einhergehenden soziökonomischen Restrukturierungsleistungen so weit fortgeschritten und gefestigt sind, dass sie die Handlungsbedingungen und -orientierungen innerhalb des Sektors und dessen weitere Entwicklungsdynamik prägen. Relative Stabilisierung heißt darüber hinaus, dass damit der Transformationsprozess an einem solchen Umschlagpunkt noch nicht zu Ende und abgeschlossen sein muss. Angesichts der oft anhaltenden technologischen Dynamiken muss die Stabilisierung einer neuen sektoralen Strukturation nicht zwingend in eine neue Phase der Kontinuität mit dann nur noch geringen Modifikationen und Feinjustierungen münden. Sie kann als temporäre Stabilisierung auch den Ausgangspunkt einer weiteren Runde gradueller Transformation bilden, die den Sektor aufs Neue signifikant verändert. Ob das so ist - das ist eine der vielen empirischen Fragen, die am konkreten Fall durchgespielt und beantwortet werden müssen. 


\section{Literatur}

Bandelow, N. (1999). Lernende Politik. Advocacy-Koalitionen und politischer Wandel am Beispiel der Gentechnologiepolitik. Berlin: edition sigma.

Barben, D. (2007). Politische Ökonomie der Biotechnologie. Innovation und gesellschaftlicher Wandel im internationalen Vergleich. Frankfurt a. M.: Campus.

Bender, G. (2006). Technologieentwicklung als Institutionalisierungsprozess. Zur Entstehung einer soziotechnischen Welt. Berlin: edition sigma.

Beyer, J. (2006). Pfadabhängigkeit. Über institutionelle Kontinuität, anfällige Stabilität und fundamentalen Wandel. Frankfurt a. M.: Campus.

Braun-Thürmann, H. (2005). Innovation. Bielefeld: Transcript.

Bresnahan, T. F., \& Malerba, F. (1999). Industrial dynamics and the evolution of firms' and Nations' competitive capabilities in the world computer industry. In D. C. Mowery \& R. R. Nelson (Hrsg.), Sources of industrial leadership. Studies of seven industries (S. 79-132). Cambridge: Cambridge University Press.

Bundesverband Musikindustrie. (Hrsg.). (2010). Jahreswirtschaftsbericht 2009. Berlin: Bundesverband Musikindustrie.

Burkart, P., \& McCourt, T. (2006). Digital music wars. Ownership and control of the celestial jukebox. Oxford: Rowman \& Littlefield.

Campbell, J. L. (2004). Institutional change and globalization. Princeton: Princeton University Press.

Campbell, J. L. (2006). What's new? General patterns of planned macro-institutional change. In J. Hage \& M. Meeus (Hrsg.), Innovation, science, and institutional change (S. 505-524). Oxford: Oxford University Press.

Currah, A. (2006). Hollywood versus the internet: The media and entertainment industries in a digital and networked economy. Journal of Economic Geography, 6, 439-468.

Currah, A. (2009). What's happening to our news. An investigation into the likely impact of the digital revolution on the economics of news publishing in the UK. Oxford: Reuters Institute.

David, P. A. (1985). Clio and the economics of QWERTY. American Economic Review, 75, 332-337.

DiMaggio, P. J., \& Powell, W. W. (1983). The iron cage revisited: Institutional isomorphism and collective rationality in organizational fields. American Sociological Review, 48, 147-160.

Djelic, M.-L., \& Quack, S. (Hrsg.). (2003). Globalization and institutions. Redefining the rules of the economic game. Cheltenham: Edward Elgar.

Djelic, M.-L., \& Quack, S. (2007). Overcoming path dependency: Path generation in open systems. Theory and Society, 36, 161-186.

Dolata, U. (1992). Weltmarktorientierte Modernisierung. Die ökonomische Regulierung des wissenschaftich-technischen Umbruchs in der Bundesrepublik. Frankfurt a. M.: Campus.

Dolata, U. (1996). Politische Ökonomie der Gentechnik. Konzernstrategien, Forschungsprogramme, Technologiewettläufe. Berlin: edition sigma.

Dolata, U. (2003). Unternehmen Technik. Akteure, Interaktionsmuster und strukturelle Kontexte der Technikentwicklung: Ein Theorierahmen. Berlin: edition sigma.

Dolata, U. (2007). 25 Jahre kommerzielle Gentechnik. Blätter für deutsche und internationale Politik, 11, 1395-1397.

Dolata, U. (2008a). Technologische Innovationen und sektoraler Wandel. Eingriffstiefe, Adaptionsfähigkeit, Transformationsmuster: Ein analytischer Ansatz. Zeitschrift für Soziologie, 37, 44-61.

Dolata, U. (2008b). Das Internet und die Transformation der Musikindustrie: Rekonstruktion und Erklärung eines unkontrollierten Wandels. Berliner Journal für Soziologie, 18, 344-369.

Dolata, U. (2009). Technological innovations and sectoral change. Transformative capacity, adaptability, patterns of change. An analytical framework. Research Policy, 38, 1066-1076. 
Dosi, G., Freeman, C., Nelson, R., Silverberg, G., \& Soete, L. (Hrsg.). (1988). Technical change and economic theory. London: Pinter.

Drews, J. (1999). In quest of tomorrow's medicines. New York: Springer.

e-business watch. (Hrsg.). (2005a). ICT and electronic business in the automotive industry. ICT adoption and e-business activity in 2005. Berlin: European Commission.

e-business watch. (Hrsg.). (2005b). ICT and electronic business in the publishing \& printing industry. Key issues and case studies. Luxembourg: European Commission.

Flowers, S. (2008). Harnessing the hackers: The emergence and exploitation of outlaw innovation. Research Policy, 37, 177-193.

Freeman, C. (1994). The economics of technical change. Cambridge Journal of Economics, 18, 463-514.

Freeman, C., \& Perez, C. (1988). Structural crises of adjustment, business cycles and investment behaviour. In G. Dosi, C. Freeman, R. Nelson, G. Silverberg, \& L. Soete (Hrsg.), Technical change and economic theory (S.38-66). London: Pinter.

Geels, F., \& Kemp, R. (2007). Dynamics in socio-technical systems: Typology of change processes and contrasting case studies. Technology in Society, 29, 441-455.

Geels, F. W., \& Schot, J. (2007). Typology of sociotechnical transition pathways. Research Policy, 36, 399-417.

Greenwood, R., \& Hinings, C. R. (1996). Understanding radical organizational change: Bringing together the old and the new institutionalism. The Academy of Management Review, 21, 1022-1054.

Hall, P. A., \& Thelen, K. (2009). Institutional change in varieties of capitalism. Socio-Economic Review, 7, 7-34.

Hannan, M. T., \& Freeman, J. (1984). Structural inertia and organizational change. American Sociological Review, 49, 149-164.

Henderson, R., \& Clark, K. B. (1990). Architectural innovation: The reconfiguration of existing product technologies and the failure of existing firms. Administrative Science Quarterly, 35, 9-30.

Henderson, R., Orsenigo, L., \& Pisano, G. P. (1999). The pharmaceutical industry and the revolution in molecular biology: Interactions among scientific, institutional, and organizational change. In D. C. Mowery \& R. R. Nelson (Hrsg.), Sources of industrial leadership. Studies of seven industries (S. 267-311). Cambridge: Cambridge University Press.

Heng, S. (2006). Medienwirtschaft vor größtem Umbruch seit Gutenberg. Der Medienkonsument auf dem Weg zum Medienmacher. E-conomics, 59, v. 12.9.2006, 1-10.

IFPI. (Hrsg.). (2009). Digital music report 2009. London: IFPI.

IFPI. (Hrsg.). (2011). Digital music report 2011. London: IFPI.

Kemp, R., Rip, A., \& Schot, J. (2001) Constructing transition paths through the management of niches. In R. Garud \& P. Karnoe (Hrsg.), Path dependence and creation (S. 269-299). London: Lawrence Erlbaum.

Kenney, M., \& Curry, J. (2001). The internet and the personal computer value chain. In BRIEIGCC e-conomy project (Hrsg.), Tracking a transformation. E-commerce and the terms of competition in industries (S. 151-177). Washington: Brookings Institution Press.

King, R. (2007). The regulatory state in an age of governance. Soft words and big sticks. Hampshire: Palgrave MacMillan.

Kitschelt, H. (1991). Industrial governance structures, innovation strategies, and the case of Japan: Sectoral or cross-national comparative analysis? International Organization, 4, 453-493.

Krasner, S. D. (1988). Sovereignty: An institutional perspective. Comparative Political Studies, 21, 66-94.

Leblebici, H., Salancik, G. R., Copay, A., \& King, T. (1991). Institutional change and the transformation of interorganizational fields: An organizational history of the U.S. radio broadcasting industry. Administrative Science Quarterly, 36, 333-363. 
van Lente, H., \& Rip, A. (1998). The rise of membrane technology: From rhetorics to social reality. Social Studies of Science, 28, 221-254.

Levinthal, D. A. (1998). The slow pace of rapid technological change: Gradualism and punctuation in technological change. Industrial and Corporate Change, 7, 217-247.

Mahoney, J., \& Thelen, K. (2010). A theory of gradual institutional change. In J. Mahoney \& K. Thelen (Hrsg.), Explaining institutional change: Ambiguity, agency, and power (S.1-37). Cambridge: Cambridge University Press.

Mautz, R., Byzio, A., \& Rosenbaum, W. (2008). Auf dem Weg zur Energiewende. Die Entwicklung der Stromproduktion aus erneuerbaren Energien in Deutschland. Göttingen: Universitätsverlag Göttingen.

Mayntz, R. (2009). The changing governance of large technical infrastructure systems. In R. Mayntz, Über Governance. Institutionen und Prozesse politischer Regelung (S.121-150). Frankfurt a. M.: Campus.

Mokyr, J. (2002). Innovation in an historical perspective: Tales of technology and evolution. In B. Steil, D. G. Victor, \& R. R. Nelson (Hrsg.), Technological innovation and economic performance (S. 23-46). Princeton: Princeton University Press.

North, D. C. (1990). Institutions, institutional change and economic performance. Cambridge: Cambridge University Press.

OECD. (Hrsg.). (2010). The evolution of news and the internet. OECD: DSTI/ICCP/IE(2009)14/FINAL.

Peitz, M., \& Waelbroek, P. (2006). Digital music. In G. Illing \& M. Peitz (Hrsg.), Industrial organization and the digital economy (S.71-144). Cambridge: MIT Press.

Pempel, T. J. (1998). Regime shift: comparative dynamics of the Japanese political economy. Ithaca: Cornell University Press.

Pierson, P. (2000). Not just what, but when: Timing and sequence in political processes. Studies in American Political Development, 14, 72-92.

Praetorius, B., Bauknecht, D., Cames, M., Fischer, C., Pehnt, M., Schumacher, K., \& Voß, J. P. (2008). Innovation for sustainable electricity systems. Exploring the dynamics of energy transitions. Dordrecht: Springer.

Quack, S. (2005). Zum Werden und Vergehen von Institutionen - Vorschläge für eine dynamische Governanceanalyse. In G. F. Schuppert (Hrsg.), Governance-Forschung. Vergewisserung über Stand und Entwicklungslinien (S. 346-370). Baden-Baden: Nomos.

RIAA. (2010). 2009 year-end shipment statistics. New York: RIAA.

Riehm, U. et al. (2003). E-Commerce in Deutschland. Eine kritische Bestandsaufnahme zum elektronischen Handel. Berlin: edition sigma.

Rip, A., \& Kemp, R. (1998). Technological change. In S. Rayner \& E. L. Malone (Hrsg.), Human choice and climate change. Volume two: Resources and technology (S. 328-399). Columbus: Batelle Press.

Rodriguez, T., von Rothkirch, M., \& Heinz, O. (Hrsg.). (2007). www.musikverkaufen.de. Die digitale Musikwirtschaft. München: Musikmarkt.

Roijakkers, N., \& Hagedoorn, J. (2006). Inter-firm partnering in pharmaceutical biotechnology since 1975: Trends, patterns, and networks. Research Policy, 35, 431-446.

Schenek, M. (1995). Das Gentechnikrecht der Europäischen Gemeinschaft. Gemeinschaftliche Biotechnologiepolitik und Gentechnikregulierung. Berlin: Duncker \& Humblot.

Schneider, V. (2001). Die Transformation der Telekommunikation: Vom Staatsmonopol zum globalen Markt (1800-2000). Frankfurt a. M.: Campus.

Schrape, J.-F. (2010). Neue Demokratie im Netz? Eine Kritik an den Visionen der Informationsgesellschaft. Bielefeld: Transcript.

Schrape, J.-F. (2011). Der Wandel des Buchhandels durch Digitalisierung und Internet. SOI Discussion paper 2011-01. Stuttgart: Stuttgarter Beiträge zur Organisations- und Innovationsforschung. 
Smith, A., Stirling, A., \& Berkhout, F. (2005). The governance of sustainable socio-technical transitions. Research Policy, 34, 1491-1510.

Streeck, W. (2009). Re-forming capitalism. Institutional change in the German political economy. Oxford: Oxford University Press.

Streeck, W., \& Thelen, K. (2005). Introduction: Institutional change in advanced political economies. In W. Streeck \& K. Thelen (Hrsg.), Beyond continuity. Institutional change in advanced political economies (S. 1-39). Oxford: Oxford University Press.

Thelen, K. (2003). How institutions evolve: Insights from comparative historical analysis. In J. Mahoney \& D. Rueschemeyer (Hrsg.), Comparative historical analysis in the social sciences (S. 208-240). Cambridge: Cambridge University Press.

Tschmuck, P. (2006). Creativity and innovation in the music industry. Springer: Dordrecht.

Voß, J.-P., \& Bauknecht, D. (2007). Der Einfluss von Technik auf Governance-Innovationen: Regulierung zur gemeinsamen Netznutzung in Infrastruktursystemen. In U. Dolata \& R. Werle (Hrsg.), Gesellschaft und die Macht der Technik. Sozioökonomischer und institutioneller Wandel durch Technisierung (S. 109-131). Frankfurt a. M.: Campus.

Walgenbach, P., \& Meyer, R. R. (2008). Neoinstitutionalistische Organisationstheorie. Stuttgart: Kohlhammer.

Werle, R. (1990). Telekommunikation in der Bundesrepublik. Expansion, Differenzierung, Transformation. Frankfurt a. M.: Campus.

Werle, R. (2005). Institutionelle Analyse technischer Innovation. Kölner Zeitschrift für Soziologie und Sozialpsychologie, 57, 308-332.

Ulrich Dolata, geb. 1959. Dr. rer. pol., Professor für Organisations- und Innovationssoziologie am Institut für Sozialwissenschaften der Universität Stuttgart. Vorstandsmitglied der Sektion Wissenschafts- und Technikforschung der Deutschen Gesellschaft für Soziologie. Forschungsschwerpunkte: Sozialwissenschaftliche Technik- und Innovationsforschung, Organisationaler und institutioneller Wandel, Politische Ökonomie des Internet. Ausgewählte Veröffentlichungen: Wandel durch Technik. Eine Theorie soziotechnischer Transformation, 2011; (mit R. Werle, Hrsg.) Gesellschaft und die Macht der Technik, 2007; Technological innovations and sectoral change. Transformative capacity, adaptability, patterns of change: An analytical framework, in: Research Policy 38, Heft 6; Das Internet und die Transformation der Musikindustrie. Rekonstruktion und Erklärung eines unkontrollierten sektoralen Wandels, in: Berliner Journal für Soziologie 18, Heft 3. 